\title{
Iterative approximation of common element of solution sets of various nonlinear operator problems
}

\section{Atid Kangtunyakarn*}

\author{
"Correspondence: \\ beawrock@hotmail.com \\ Department of Mathematics, \\ Faculty of Science, King Mongkut's \\ Institute of Technology Ladkrabang, \\ Bangkok 10520, Thailand
}

\begin{abstract}
In this paper, we prove strong convergence theorem for finding a common element of the set of fixed point of a finite family of nonexpansive mappings and a finite family of $\kappa_{i}$-strictly pseudocontractive mappings and the set of a finite family of the set of solution of equilibrium problems by using the new mapping generated by a finite family of nonexpansive mappings and a finite family of $\boldsymbol{\kappa}_{i}$-strictly pseudocontractive mappings and a sequences of positive real numbers. Furthermore, by using our main result, we obtain two interesting theorems involving variational inequality problems and variational inclusion problems. In the last section, we give numerical examples to support our main results.
\end{abstract}

Keywords: nonexpansive mapping; strictly pseudocontractive mapping; equilibrium problem; variational inequality problem; variational inclusion problem

\section{Introduction}

Let $H$ be a real Hilbert space and $C$ be a nonempty closed convex subset of $H$. A self mapping $f: C \rightarrow C$ is a contraction on $C$ if there exists a constant $k \in[0,1)$ such that $\|f(x)-f(y)\| \leq k\|x-y\|, \forall x, y \in C$. Let $T: C \rightarrow C$ be a mapping, a point $x \in C$ is called a fixed point of $T$ if and only if $T x=x$. In this paper, we use $F(T)$ to denote the set of fixed point of $T$. Recall the following definitions.

Definition 1.1 A mapping $T: C \rightarrow C$ is called nonexpansive if and only if for all $x, y \in C$,

$$
\|T x-T y\| \leq\|x-y\| .
$$

Definition 1.2 A mapping $T: C \rightarrow C$ is called $\kappa$-strictly pseudocontractive [1] if and only if there exists a constant $\kappa \in[0,1)$ such that for all $x, y \in C$,

$$
\|T x-T y\|^{2} \leq\|x-y\|^{2}+\kappa\|(I-T) x-(I-T) y\|^{2} .
$$

For such case, $T$ is also said to be a $\kappa$-strictly pseudo contraction.

Note that the class of $\kappa$-strict pseudo-contractions strictly includes the class of nonexpansive mappings, that is $T$ is nonexpansive if and only if $T$ is 0 -strict pseudocontractive.

(2013 Kangtunyakarn; licensee Springer. This is an Open Access article distributed under the terms of the Creative Commons Attribution License (http://creativecommons.org/licenses/by/2.0), which permits unrestricted use, distribution, and reproduction in any medium, provided the original work is properly cited. 
Let $F: C \times C \rightarrow \mathbb{R}$ be a bifunction. The equilibrium problem for $F$ is to determine its equilibrium points, i.e., the set

$$
E P(F)=\{x \in C: F(x, y) \geq 0, \forall y \in C\} .
$$

Given $T: C \rightarrow H$, let $F(x, y)=\langle T x, y-x\rangle$ for all $x, y \in C$. Then $z \in E P(F)$ if and only if $\langle T z, y-z\rangle \geq 0$ for all $y \in C$, that is, $z$ is a solution of the variational inequality.

Equilibrium problems, which were introduced in [2] in 1994, have had a great impact and influence in the development of several branches of pure and applied sciences. Numerous problems in physics, minimization problems, Nash equilibria in noncooperative games, optimization and economics reduce to find a solution of $E P(F)$ (see, for example, [2-4]). Some methods have been proposed to solve the equilibrium problem (see, for example, [5-7]).

In 2007, Takahashi and Takahashi [8] proved the following theorem.

Theorem 1.1 Let $C$ be a nonempty closed convex subset of $H$. Let $F$ be a bifunction from $C \times C$ to $\mathbb{R}$ satisfying

(A1) $F(x, x)=0, \forall x \in C$;

(A2) $F$ is monotone, i.e., $F(x, y)+F(y, x) \leq 0, \forall x, y \in C$;

(A3) $\forall x, y, z \in C$,

$$
\lim _{t \rightarrow 0^{+}} F(t z+(1-t) x, y) \leq F(x, y)
$$

(A4) $\forall x \in C, y \mapsto F(x, y)$ is convex and lower semicontinuous;

and let $S$ be a nonexpansive mapping of $C$ into $H$ such that $F(S) \cap E P(G) \neq \emptyset$. Let $f$ be a contraction of $H$ into itself, and let $\left\{x_{n}\right\}$ and $\left\{u_{n}\right\}$ be sequences generated by $x_{1} \in H$ and

$$
\begin{aligned}
& F\left(u_{n}, y\right)+\frac{1}{r_{n}}\left\langle y-u_{n}, u_{n}-x_{n}\right\rangle \geq 0, \quad \forall y \in C, \\
& x_{n+1}=\alpha_{n} f\left(x_{n}\right)+\left(1-\alpha_{n}\right) S u_{n}
\end{aligned}
$$

for all $n \in N$, where $\left\{\alpha_{n}\right\} \subset[0,1]$ and $\left\{r_{n}\right\} \subset(0,1)$ satisfy (C1)-(C3) as follows:

(C1) $\alpha_{n} \rightarrow 0$;

(C2) $\sum_{n=0}^{\infty} \alpha_{n}=\infty$;

(C3) either $\sum_{n=0}^{\infty}\left|\alpha_{n+1}-\alpha_{n}\right|<\infty$ or $\lim _{n \rightarrow \infty} \frac{\alpha_{n+1}}{\alpha_{n}}=1$, and $\liminf _{n \rightarrow \infty} r_{n}>0$ and $\sum_{n=1}^{\infty}\left|r_{n+1}-r_{n}\right|<\infty$.

Then $\left\{x_{n}\right\}$ and $\left\{u_{n}\right\}$ converge strongly to $z \in F(S) \cap E P(F)$, where $z=P_{F(S) \cap E P(F)} f(z)$.

In 2010, Kangtunyakarn and Suantai [9] proved the strong convergence theorem by using the $S$-mapping generated by a finite family of strictly pseudocontractive mappings and a finite family of real number as follows.

Theorem 1.2 Let $H$ be a Hilbert space, let $f$ be an $\alpha$-contraction on $H$, and let $A$ be a strongly positive linear bounded self-adjoint operator with coefficient $\bar{\gamma}>0$. Assume that $0<\gamma<\frac{\bar{\gamma}}{\alpha}$. Let $\left\{T_{i}\right\}_{i=1}^{N}$ be a finite family of $\kappa_{i}$-strictly pseudo contraction of $H$ into itself for some $\kappa_{i} \in[0,1)$ and $\kappa=\max \left\{\kappa_{i}: i=1,2, \ldots, N\right\}$ with $\bigcap_{i=1}^{N} F\left(T_{i}\right) \neq \emptyset$. Let $S_{n}$ be the $S$ mappings generated by $T_{1}, T_{2}, \ldots, T_{N}$ and $\alpha_{1}^{(n)}, \alpha_{2}^{(n)}, \ldots, \alpha_{N}^{(n)}$, where $\alpha_{j}^{(n)}=\left(\alpha_{1}^{n, j}, \alpha_{2}^{n, j}, \alpha_{3}^{n, j}\right) \in$ 
$I \times I \times I, I=[0,1], \alpha_{1}^{n, j}+\alpha_{2}^{n, j}+\alpha_{3}^{n, j}=1$ and $\kappa<a \leq \alpha_{1}^{n, j}, \alpha_{3}^{n, j} \leq b<1$ for all $j=1,2, \ldots, N-1$, $\kappa<c \leq \alpha_{1}^{n, N} \leq 1, \kappa \leq \alpha_{3}^{n, N} \leq d<1, \kappa \leq \alpha_{2}^{n, j} \leq e<1$ for all $j=1,2, \ldots, N$. For a point $u \in H$ and $x_{1} \in H$, let $\left\{x_{n}\right\}$ and $\left\{y_{n}\right\}$ be the sequences defined iteratively by

$$
\left\{\begin{array}{l}
y_{n}=\beta_{n} x_{n}+\left(1-\beta_{n}\right) S_{n} x_{n}, \\
x_{n+1}=\alpha_{n} \gamma\left(a_{n} u+\left(1-a_{n}\right) f\left(x_{n}\right)\right)+\left(I-\alpha_{n} A\right) y_{n}, \quad n \geq 1,
\end{array}\right.
$$

where $\left\{\beta_{n}\right\},\left\{\alpha_{n}\right\}$ and $\left\{a_{n}\right\}$ are sequences in $[0,1]$. Assume that the following conditions hold:

(i) $\lim _{n \rightarrow \infty} \alpha_{n}=0, \sum_{n=1}^{\infty} \alpha_{n}=\infty$ and $\lim _{n \rightarrow \infty} a_{n}=0$;

(ii) $\sum_{n=1}^{\infty}\left|\alpha_{1}^{n+1, j}-\alpha_{1}^{n, j}\right|<\infty, \sum_{n=1}^{\infty}\left|\alpha_{3}^{n+1, j}-\alpha_{3}^{n, j}\right|<\infty$ for all $j \in\{1,2,3, \ldots, N\}$ and $\sum_{n=1}^{\infty}\left|\alpha_{n+1}-\alpha_{n}\right|<\infty, \sum_{n=1}^{\infty}\left|\beta_{n+1}-\beta_{n}\right|<\infty$ and $\sum_{n=1}^{\infty}\left|a_{n+1}-a_{n}\right|<\infty$;

(iii) $0 \leq \kappa \leq \beta_{n}<\theta<1$ for all $n \geq 1$ for some $\theta \in(0,1)$.

Then both $\left\{x_{n}\right\}$ and $\left\{y_{n}\right\}$ strongly converge to $q \in \bigcap_{i=1}^{N} F\left(T_{i}\right)$, which solves the following variational inequality

$$
\langle\gamma f(q)-A q, p-q\rangle \leq 0, \quad \forall p \in \bigcap_{i=1}^{N} F\left(T_{i}\right) .
$$

Question Can we prove a strong convergence theorem for finding a common solution of the set of fixed point of a finite family of nonexpansive mappings and a finite family of strictly pseudocontractive mappings and a finite family of the set of solution of equilibrium problems?

Let $C$ be a nonempty closed convex subset of Hilbert space $H$. Let $\left\{T_{i}\right\}_{i=1}^{N}$ be a finite family of $\kappa_{i}$-strict pseudo-contractions of $C$ into itself, and let $\left\{S_{i}\right\}_{i=1}^{N}$ be a finite family of nonexpansive mappings of $C$ into itself. For each $n \in \mathbb{N}$ and $j=1,2, \ldots, N$, let $\alpha_{j}^{(n)}=$ $\left(\alpha_{1}^{n, j}, \alpha_{2}^{n, j}, \alpha_{3}^{n, j}\right) \in I \times I \times I$, where $I=[0,1], \alpha_{1}^{n, j}+\alpha_{2}^{n, j}+\alpha_{3}^{n, j}=1$. We define the mapping $S_{n}^{A}: C \rightarrow C$ as follows:

$$
\begin{aligned}
& U_{n, 0}=I, \\
& U_{n, 1}=S_{1}\left(\alpha_{1}^{n, 1} T_{1} U_{n, 0}+\alpha_{2}^{n, 1} U_{n, 0}+\alpha_{3}^{n, 1} I\right), \\
& U_{n, 2}=S_{2}\left(\alpha_{1}^{n, 2} T_{2} U_{n, 1}+\alpha_{2}^{n, 2} U_{n, 1}+\alpha_{3}^{n, 2} I\right), \\
& U_{n, 3}=S_{3}\left(\alpha_{1}^{n, 3} T_{3} U_{n, 2}+\alpha_{2}^{n, 3} U_{n, 2}+\alpha_{3}^{n, 3} I\right), \\
& \vdots \\
& U_{n, N-1}=S_{N-1}\left(\alpha_{1}^{n, N-1} T_{N-1} U_{n, N-2}+\alpha_{2}^{n, N-1} U_{n, N-2}+\alpha_{3}^{n, N-1} I\right), \\
& S_{n}^{A}=U_{n, N}=S_{N}\left(\alpha_{1}^{n, N} T_{N} U_{n, N-1}+\alpha_{2}^{n, N} U_{n, N-1}+\alpha_{3}^{n, N} I\right) .
\end{aligned}
$$

In Lemma 2.8, under suitable conditions of the real sequences $\left\{\alpha_{1}^{n, j}\right\},\left\{\alpha_{2}^{n, j}\right\}$ and $\left\{\alpha_{3}^{n, j}\right\}$ for every $j=1,2, \ldots, N$, we show that $F\left(S_{n}^{A}\right)=\bigcap_{i=1}^{N} F\left(S_{i}\right) \cap \bigcap_{i=1}^{N} F\left(T_{i}\right)$ and $S_{n}^{A}$ is a nonexpansive mapping.

In this paper, motivated by the ongoing research and Theorems 1.1 and 1.2, we prove strong convergence theorem for finding a common solution of the set of fixed point of a finite family of nonexpansive mappings and a finite family of strictly pseudocontractive 
mappings and a finite family of the set of solution of equilibrium problems by using the mapping defined by (1.4). Furthermore, in the last section, we prove two interesting theorems involving a finite family of the set of solutions of variational inequality problem and variational inclusion problem. In the last section, we give numerical examples to support our main results.

\section{Preliminaries}

In this section, we need the following lemmas to prove our main result. Let $C$ be a closed convex subset of a real Hilbert space $H$, let $P_{C}$ be the metric projection of $H$ onto $C$, i.e., for $x \in H, P_{C} x$ satisfies the property

$$
\left\|x-P_{C} x\right\|=\min _{y \in C}\|x-y\| .
$$

The following characterizes the projection $P_{C}$.

Lemma 2.1 (See [10]) Given $x \in H$ and $y \in C$. Then $P_{C} x=y$ if and only if the following inequality holds

$$
\langle x-y, y-z\rangle \geq 0, \quad \forall z \in C
$$

Lemma 2.2 (See [11]) Let $\left\{s_{n}\right\}$ be a sequence of nonnegative real numbers satisfying

$$
s_{n+1}=\left(1-\alpha_{n}\right) s_{n}+\alpha_{n} \beta_{n}, \quad \forall n \geq 0,
$$

where $\left\{\alpha_{n}\right\},\left\{\beta_{n}\right\}$ satisfy the conditions

$$
\begin{aligned}
& \text { (1) } \quad\left\{\alpha_{n}\right\} \subset[0,1], \quad \sum_{n=1}^{\infty} \alpha_{n}=\infty ; \\
& \text { (2) } \quad \limsup _{n \rightarrow \infty} \beta_{n} \leq 0 \quad \text { or } \quad \sum_{n=1}^{\infty}\left|\alpha_{n} \beta_{n}\right|<\infty .
\end{aligned}
$$

Then $\lim _{n \rightarrow \infty} s_{n}=0$.

Lemma 2.3 (See [12]) Let $\left\{s_{n}\right\}$ be a sequence of nonnegative real numbers satisfying

$$
s_{n+1}=\left(1-\alpha_{n}\right) s_{n}+\delta_{n}, \quad \forall n \geq 0,
$$

where $\left\{\alpha_{n}\right\}$ is a sequence in $(0,1)$ and $\left\{\delta_{n}\right\}$ is a sequence such that

$$
\begin{aligned}
& \text { (1) } \sum_{n=1}^{\infty} \alpha_{n}=\infty ; \\
& \text { (2) } \limsup _{n \rightarrow \infty} \frac{\delta_{n}}{\alpha_{n}} \leq 0 \quad \text { or } \quad \sum_{n=1}^{\infty}\left|\delta_{n}\right|<\infty \text {. }
\end{aligned}
$$

Then $\lim _{n \rightarrow \infty} s_{n}=0$. 
Lemma 2.4 (See [13]) Let $C$ be a nonempty closed convex subset of a real Hilbert space $H$, and let $S: C \rightarrow C$ be a self-mapping of $C$. If $S$ is a $\kappa$-strict pseudo-contraction mapping, then $S$ satisfies the Lipschitz condition

$$
\|S x-S y\| \leq \frac{1+\kappa}{1-\kappa}\|x-y\|, \quad \forall x, y \in C .
$$

For solving the equilibrium problem for a bifunction $F: C \times C \rightarrow \mathbb{R}$, let us assume that $F$ satisfies the following conditions:

(A1) $F(x, x)=0, \forall x \in C$;

(A2) $F$ is monotone, i.e., $F(x, y)+F(y, x) \leq 0, \forall x, y \in C$;

(A3) $\forall x, y, z \in C$,

$$
\lim _{t \rightarrow 0^{+}} F(t z+(1-t) x, y) \leq F(x, y)
$$

(A4) $\forall x \in C, y \mapsto F(x, y)$ is convex and lower semicontinuous.

The following lemma appears implicitly in [2].

Lemma 2.5 (See [2]) Let $C$ be a nonempty closed convex subset of $H$, and let $F$ be a bifunction of $C \times C$ into $\mathbb{R}$ satisfying (A1)-(A4). Let $r>0$ and $x \in H$. Then there exists $z \in C$ such that

$$
F(z, y)+\frac{1}{r}\langle y-z, z-x\rangle
$$

for all $y \in C$.

Lemma 2.6 (See [14]) Assume that $F: C \times C \rightarrow \mathbb{R}$ satisfies (A1)-(A4). For $r>0$ and $x \in H$, define a mapping $T_{r}: H \rightarrow C$ as follows:

$$
T_{r}(x)=\left\{z \in C: F(z, y)+\frac{1}{r}\langle y-z, z-x\rangle \geq 0, \forall y \in C\right\}
$$

for all $z \in H$. Then the following hold:

(1) $T_{r}$ is single-valued;

(2) $T_{r}$ is firmly nonexpansive, i.e.,

$$
\left\|T_{r}(x)-T_{r}(y)\right\|^{2} \leq\left\langle T_{r}(x)-T_{r}(y), x-y\right\rangle, \quad \forall x, y \in H
$$

(3) $F\left(T_{r}\right)=E P(F)$;

(4) $E P(F)$ is closed and convex.

Lemma 2.7 (See [15]) Let E be a uniformly convex Banach space, $C$ be a nonempty closed convex subset of $E$, and $S: C \rightarrow C$ be a nonexpansive mapping. Then $I-S$ is demi-closed at zero.

Definition 2.1 Let $C$ be a nonempty convex subset of real Hilbert space. Let $\left\{T_{i}\right\}_{i=1}^{N}$ be a finite family of $\kappa_{i}$-strict pseudo-contractions of $C$ into itself, and let $\left\{S_{i}\right\}_{i=1}^{N}$ be a finite family of nonexpansive mappings of $C$ into itself. For each $j=1,2, \ldots, N$, let $\alpha_{j}=\left(\alpha_{1}^{j}, \alpha_{2}^{j}, \alpha_{3}^{j}\right) \in$ 
$I \times I \times I$, where $I \in[0,1]$ and $\alpha_{1}^{j}+\alpha_{2}^{j}+\alpha_{3}^{j}=1$. We define the mapping $S^{A}: C \rightarrow C$ as follows:

$$
\begin{aligned}
& U_{0}=I \\
& U_{1}=S_{1}\left(\alpha_{1}^{1} T_{1} U_{0}+\alpha_{2}^{1} U_{0}+\alpha_{3}^{1} I\right) \\
& U_{2}=S_{2}\left(\alpha_{1}^{2} T_{2} U_{1}+\alpha_{2}^{2} U_{1}+\alpha_{3}^{2} I\right) \\
& U_{3}=S_{3}\left(\alpha_{1}^{3} T_{3} U_{2}+\alpha_{2}^{3} U_{2}+\alpha_{3}^{3} I\right) \\
& \vdots \\
& U_{N-1}=S_{N-1}\left(\alpha_{1}^{N-1} T_{N-1} U_{N-2}+\alpha_{2}^{N-1} U_{N-2}+\alpha_{3}^{N-1} I\right), \\
& S^{A}=U_{N}=S_{N}\left(\alpha_{1}^{N} T_{N} U_{N-1}+\alpha_{2}^{N} U_{N-1}+\alpha_{3}^{N} I\right) .
\end{aligned}
$$

This mapping is called the $S^{A}$-mapping generated by $S_{1}, S_{2}, \ldots, S_{N}, T_{1}, T_{2}, \ldots, T_{N}$ and $\alpha_{1}, \alpha_{2}, \ldots, \alpha_{N}$.

Lemma 2.8 Let C be a nonempty closed convex subset of a real Hilbert space. Let $\left\{T_{i}\right\}_{i=1}^{N}$ be a finite family of $\kappa_{i}$-strict pseudo-contractions of $C$ into itself, and let $\left\{S_{i}\right\}_{i=1}^{N}$ be a finite family of nonexpansive mappings of $C$ into itself with $\bigcap_{i=1}^{N} F\left(S_{i}\right) \cap \bigcap_{i=1}^{N} F\left(T_{i}\right) \neq \emptyset$ and $\kappa=$ $\max \left\{\kappa_{i}: i=1,2, \ldots, N\right\}$, and let $\alpha_{j}=\left(\alpha_{1}^{j}, \alpha_{2}^{j}, \alpha_{3}^{j}\right) \in I \times I \times I, j=1,2,3, \ldots, N$, where $I=[0,1]$, $\alpha_{1}^{j}+\alpha_{2}^{j}+\alpha_{3}^{j}=1, \alpha_{1}^{j}, \alpha_{3}^{j} \in(\kappa, 1)$ for all $j=1,2, \ldots, N-1$ and $\alpha_{1}^{N} \in(\kappa, 1], \alpha_{3}^{N} \in[\kappa, 1), \alpha_{2}^{j} \in(\kappa, 1)$ for all $j=1,2, \ldots, N$. Let $S^{A}$ be the $S^{A}$-mapping generated by $S_{1}, S_{2}, \ldots, S_{N}, T_{1}, T_{2}, \ldots, T_{N}$ and $\alpha_{1}, \alpha_{2}, \ldots, \alpha_{N}$. Then $F\left(S^{A}\right)=\bigcap_{i=1}^{N} F\left(S_{i}\right) \cap \bigcap_{i=1}^{N} F\left(T_{i}\right)$, and $S^{A}$ is a nonexpansive mapping.

Proof It is easy to see that $\bigcap_{i=1}^{N} F\left(S_{i}\right) \cap \bigcap_{i=1}^{N} F\left(T_{i}\right) \subseteq F\left(S^{A}\right)$. Let $x_{0} \in F\left(S^{A}\right)$ and $x^{*} \in$ $\bigcap_{i=1}^{N} F\left(S_{i}\right) \cap \bigcap_{i=1}^{N} F\left(T_{i}\right)$. Then we have

$$
\begin{aligned}
\left\|S^{A} x_{0}-x^{*}\right\|^{2}= & \left\|S_{N}\left(\alpha_{1}^{N} T_{N} U_{N-1} x_{0}+\alpha_{2}^{N} U_{N-1} x_{0}+\alpha_{3}^{N} x_{0}\right)-x^{*}\right\|^{2} \\
\leq & \left\|\alpha_{1}^{N} T_{N} U_{N-1} x_{0}+\alpha_{2}^{N} U_{N-1} x_{0}+\alpha_{3}^{N} x_{0}-x^{*}\right\|^{2} \\
= & \left\|\alpha_{1}^{N}\left(T_{N} U_{N-1} x_{0}-x^{*}\right)+\alpha_{2}^{N}\left(U_{N-1} x_{0}-x^{*}\right)+\alpha_{3}^{N}\left(x_{0}-x^{*}\right)\right\|^{2} \\
= & \alpha_{1}^{N}\left\|T_{N} U_{N-1} x_{0}-x^{*}\right\|^{2}+\alpha_{2}^{N}\left\|U_{N-1} x_{0}-x^{*}\right\|^{2}+\alpha_{3}^{N}\left\|x_{0}-x^{*}\right\|^{2} \\
& -\alpha_{1}^{N} \alpha_{2}^{N}\left\|T_{N} U_{N-1} x_{0}-U_{N-1} x_{0}\right\|^{2}-\alpha_{1}^{N} \alpha_{3}^{N}\left\|T_{N} U_{N-1} x_{0}-x_{0}\right\|^{2} \\
& -\alpha_{2}^{N} \alpha_{3}^{N}\left\|U_{N-1} x_{0}-x_{0}\right\|^{2} \\
\leq & \alpha_{1}^{N}\left(\left\|U_{N-1} x_{0}-x^{*}\right\|^{2}+\kappa\left\|\left(I-T_{N}\right) U_{N-1} x_{0}-\left(I-T_{N}\right) x^{*}\right\|^{2}\right) \\
& +\alpha_{2}^{N}\left\|U_{N-1} x_{0}-x^{*}\right\|^{2}+\alpha_{3}^{N}\left\|x_{0}-x^{*}\right\|^{2}-\alpha_{1}^{N} \alpha_{2}^{N}\left\|T_{N} U_{N-1} x_{0}-U_{N-1} x_{0}\right\|^{2} \\
& -\alpha_{1}^{N} \alpha_{3}^{N}\left\|T_{N} U_{N-1} x_{0}-x_{0}\right\|^{2}-\alpha_{2}^{N} \alpha_{3}^{N}\left\|U_{N-1} x_{0}-x_{0}\right\|^{2} \\
= & \left(1-\alpha_{3}^{N}\right)\left\|U_{N-1} x_{0}-x^{*}\right\|^{2}+\alpha_{1}^{N}\left(\kappa-\alpha_{2}^{N}\right)\left\|\left(I-T_{N}\right) U_{N-1} x_{0}\right\|^{2} \\
& +\left(1-\left(1-\alpha_{3}^{N}\right)\right)\left\|x_{0}-x^{*}\right\|^{2}-\alpha_{1}^{N} \alpha_{3}^{N}\left\|T_{N} U_{N-1} x_{0}-x_{0}\right\|^{2} \\
& -\alpha_{2}^{N} \alpha_{3}^{N}\left\|U_{N-1} x_{0}-x_{0}\right\|^{2} \\
\leq & \left(1-\alpha_{3}^{N}\right)\left\|U_{N-1} x_{0}-x^{*}\right\|^{2}+\alpha_{1}^{N}\left(\kappa-\alpha_{2}^{N}\right)\left\|\left(I-T_{N}\right) U_{N-1} x_{0}\right\|^{2}
\end{aligned}
$$




$$
\begin{aligned}
& +\left(1-\left(1-\alpha_{3}^{N}\right)\right)\left\|x_{0}-x^{*}\right\|^{2}-\alpha_{2}^{N} \alpha_{3}^{N}\left\|U_{N-1} x_{0}-x_{0}\right\|^{2} \\
& \leq \prod_{j=N-1}^{N}\left(1-\alpha_{3}^{j}\right)\left\|U_{N-2} x_{0}-x^{*}\right\|^{2} \\
& +\left(1-\alpha_{3}^{N}\right) \alpha_{1}^{N-1}\left(\kappa-\alpha_{2}^{N-1}\right)\left\|\left(I-T_{N-1}\right) U_{N-2} x_{0}\right\|^{2} \\
& -\left(1-\alpha_{3}^{N}\right) \alpha_{2}^{N-1} \alpha_{3}^{N-1}\left\|U_{N-2} x_{0}-x_{0}\right\|^{2} \\
& +\left(1-\prod_{j=N-1}^{N}\left(1-\alpha_{3}^{j}\right)\right)\left\|x_{0}-x^{*}\right\|^{2} \\
& \leq \prod_{j=N-1}^{N}\left(1-\alpha_{3}^{j}\right)\left\|U_{N-2} x_{0}-x^{*}\right\|^{2}+\left(1-\prod_{j=N-1}^{N}\left(1-\alpha_{3}^{j}\right)\right)\left\|x_{0}-x^{*}\right\|^{2} \\
& \leq \prod_{j=N-2}^{N}\left(1-\alpha_{3}^{j}\right)\left\|U_{N-3} x_{0}-x^{*}\right\|^{2} \\
& +\prod_{j=N-1}^{N}\left(1-\alpha_{3}^{j}\right) \alpha_{1}^{N-2}\left(\kappa-\alpha_{2}^{N-2}\right)\left\|\left(I-T_{N-2}\right) U_{N-3} x_{0}\right\|^{2} \\
& -\prod_{j=N-1}^{N}\left(1-\alpha_{3}^{j}\right) \alpha_{2}^{N-2} \alpha_{3}^{N-2}\left\|U_{N-3} x_{0}-x_{0}\right\|^{2} \\
& +\left(1-\prod_{j=N-2}^{N}\left(1-\alpha_{3}^{j}\right)\right)\left\|x_{0}-x^{*}\right\|^{2} \\
& \leq \prod_{j=N-2}^{N}\left(1-\alpha_{3}^{j}\right)\left\|U_{N-3} x_{0}-x^{*}\right\|^{2}+\left(1-\prod_{j=N-2}^{N}\left(1-\alpha_{3}^{j}\right)\right)\left\|x_{0}-x^{*}\right\|^{2} \\
& \leq \prod_{j=3}^{N}\left(1-\alpha_{3}^{j}\right)\left\|U_{2} x_{0}-x^{*}\right\|^{2} \\
& +\prod_{j=4}^{N}\left(1-\alpha_{3}^{j}\right) \alpha_{1}^{3}\left(\kappa-\alpha_{2}^{3}\right)\left\|\left(I-T_{3}\right) U_{2} x_{0}\right\|^{2} \\
& -\prod_{j=4}^{N}\left(1-\alpha_{3}^{j}\right) \alpha_{2}^{3} \alpha_{3}^{3}\left\|U_{2} x_{0}-x_{0}\right\|^{2} \\
& +\left(1-\prod_{j=3}^{N}\left(1-\alpha_{3}^{j}\right)\right)\left\|x_{0}-x^{*}\right\|^{2} \\
& \leq \prod_{j=3}^{N}\left(1-\alpha_{3}^{j}\right)\left\|U_{2} x_{0}-x^{*}\right\|^{2}+\left(1-\prod_{j=3}^{N}\left(1-\alpha_{3}^{j}\right)\right)\left\|x_{0}-x^{*}\right\|^{2} \\
& \leq \prod_{j=2}^{N}\left(1-\alpha_{3}^{j}\right)\left\|U_{1} x_{0}-x^{*}\right\|^{2}+\prod_{j=3}^{N}\left(1-\alpha_{3}^{j}\right) \alpha_{1}^{2}\left(\kappa-\alpha_{2}^{2}\right)\left\|\left(I-T_{2}\right) U_{1} x_{0}\right\|^{2} \\
& -\prod_{j=3}^{N}\left(1-\alpha_{3}^{j}\right) \alpha_{2}^{2} \alpha_{3}^{2}\left\|U_{1} x_{0}-x_{0}\right\|^{2}
\end{aligned}
$$




$$
\begin{aligned}
& +\left(1-\prod_{j=2}^{N}\left(1-\alpha_{3}^{j}\right)\right)\left\|x_{0}-x^{*}\right\|^{2} \\
\leq & \prod_{j=2}^{N}\left(1-\alpha_{3}^{j}\right)\left\|U_{1} x_{0}-x^{*}\right\|^{2}+\left(1-\prod_{j=2}^{N}\left(1-\alpha_{3}^{j}\right)\right)\left\|x_{0}-x^{*}\right\|^{2} \\
\leq & \prod_{j=1}^{N}\left(1-\alpha_{3}^{j}\right)\left\|U_{0} x_{0}-x^{*}\right\|^{2} \\
& +\prod_{j=2}^{N}\left(1-\alpha_{3}^{j}\right) \alpha_{1}^{1}\left(\kappa-\alpha_{2}^{1}\right)\left\|\left(I-T_{1}\right) U_{0} x_{0}\right\|^{2} \\
& -\prod_{j=2}^{N}\left(1-\alpha_{3}^{j}\right) \alpha_{2}^{1} \alpha_{3}^{1}\left\|U_{0} x_{0}-x_{0}\right\|^{2}+\left(1-\prod_{j=1}^{N}\left(1-\alpha_{3}^{j}\right)\right)\left\|x_{0}-x^{*}\right\|^{2} \\
\leq & \left\|x_{0}-x^{*}\right\|^{2}+\prod_{j=2}^{N}\left(1-\alpha_{3}^{j}\right) \alpha_{1}^{1}\left(\kappa-\alpha_{2}^{1}\right)\left\|\left(I-T_{1}\right) x_{0}\right\|^{2} .
\end{aligned}
$$

By (2.4), we have

$$
\prod_{j=2}^{N}\left(1-\alpha_{3}^{j}\right) \alpha_{1}^{1}\left(\alpha_{2}^{1}-\kappa\right)\left\|\left(I-T_{1}\right) x_{0}\right\|^{2} \leq\left\|x_{0}-x^{*}\right\|^{2}-\left\|x_{0}-x^{*}\right\|^{2}=0,
$$

which implies that $T_{1} x_{0}=x_{0}$, that is, $x_{0} \in F\left(T_{1}\right)$. It implies that

$$
U_{1} x_{0}=S_{1}\left(\alpha_{1}^{1} T_{1} U_{0} x_{0}+\alpha_{2}^{1} U_{0} x_{0}+\alpha_{3}^{1} x_{0}\right)=S_{1} x_{0}
$$

By (2.3) and (2.5), we have

$$
\begin{aligned}
\left\|S^{A} x_{0}-x^{*}\right\|^{2} \leq & \prod_{j=2}^{N}\left(1-\alpha_{3}^{j}\right)\left\|S_{1} x_{0}-x^{*}\right\|^{2} \\
& +\prod_{j=3}^{N}\left(1-\alpha_{3}^{j}\right) \alpha_{1}^{2}\left(\kappa-\alpha_{2}^{2}\right)\left\|\left(I-T_{2}\right) U_{1} x_{0}\right\|^{2} \\
& -\prod_{j=3}^{N}\left(1-\alpha_{3}^{j}\right) \alpha_{2}^{2} \alpha_{3}^{2}\left\|U_{1} x_{0}-x_{0}\right\|^{2} \\
& +\left(1-\prod_{j=2}^{N}\left(1-\alpha_{3}^{j}\right)\right)\left\|x_{0}-x^{*}\right\|^{2} \\
\leq & \left\|x_{0}-x^{*}\right\|^{2}-\prod_{j=3}^{N}\left(1-\alpha_{3}^{j}\right) \alpha_{2}^{2} \alpha_{3}^{2}\left\|U_{1} x_{0}-x_{0}\right\|^{2} .
\end{aligned}
$$

By (2.6), we have

$$
\prod_{j=3}^{N}\left(1-\alpha_{3}^{j}\right) \alpha_{2}^{2} \alpha_{3}^{2}\left\|U_{1} x_{0}-x_{0}\right\|^{2} \leq 0
$$


It implies that

$$
x_{0}=U_{1} x_{0} .
$$

By (2.5) and (2.7), we have $x_{0} \in F\left(S_{1}\right)$. Hence, we have

$$
x_{0} \in F\left(S_{1}\right) \cap F\left(T_{1}\right) .
$$

Since $x_{0}=U_{1} x_{0}$ and (2.3), we have

$$
\begin{aligned}
\left\|S^{A} x_{0}-x^{*}\right\|^{2} \leq & \prod_{j=2}^{N}\left(1-\alpha_{3}^{j}\right)\left\|U_{1} x_{0}-x^{*}\right\|^{2} \\
& +\prod_{j=3}^{N}\left(1-\alpha_{3}^{j}\right) \alpha_{1}^{2}\left(\kappa-\alpha_{2}^{2}\right)\left\|\left(I-T_{2}\right) U_{1} x_{0}\right\|^{2} \\
& -\prod_{j=3}^{N}\left(1-\alpha_{3}^{j}\right) \alpha_{2}^{2} \alpha_{3}^{2}\left\|U_{1} x_{0}-x_{0}\right\|^{2} \\
& +\left(1-\prod_{j=2}^{N}\left(1-\alpha_{3}^{j}\right)\right)\left\|x_{0}-x^{*}\right\|^{2} \\
= & \prod_{j=2}^{N}\left(1-\alpha_{3}^{j}\right)\left\|x_{0}-x^{*}\right\|^{2} \\
& +\prod_{j=3}^{N}\left(1-\alpha_{3}^{j}\right) \alpha_{1}^{2}\left(\kappa-\alpha_{2}^{2}\right)\left\|\left(I-T_{2}\right) x_{0}\right\|^{2} \\
& +\left(1-\prod_{j=2}^{N}\left(1-\alpha_{3}^{j}\right)\right)\left\|x_{0}-x^{*}\right\|^{2} .
\end{aligned}
$$

It follows that

$$
\prod_{j=3}^{N}\left(1-\alpha_{3}^{j}\right) \alpha_{1}^{2}\left(\alpha_{2}^{2}-\kappa\right)\left\|\left(I-T_{2}\right) x_{0}\right\|^{2} \leq 0,
$$

which implies that $x_{0}=T_{2} x_{0}$, that is, $x_{0} \in F\left(T_{2}\right)$. Since $x_{0}=U_{1} x_{0}=T_{2} x_{0}$, we have

$$
U_{2} x_{0}=S_{2}\left(\alpha_{1}^{2} T_{2} U_{1} x_{0}+\alpha_{2}^{2} U_{1} x_{0}+\alpha_{3}^{2} x_{0}\right)=S_{2} x_{0} .
$$

By (2.2), we have

$$
\begin{aligned}
\left\|S^{A} x_{0}-x^{*}\right\|^{2} \leq & \prod_{j=3}^{N}\left(1-\alpha_{3}^{j}\right)\left\|U_{2} x_{0}-x^{*}\right\|^{2} \\
& +\prod_{j=4}^{N}\left(1-\alpha_{3}^{j}\right) \alpha_{1}^{3}\left(\kappa-\alpha_{2}^{3}\right)\left\|\left(I-T_{3}\right) U_{2} x_{0}\right\|^{2}
\end{aligned}
$$




$$
\begin{aligned}
& -\prod_{j=4}^{N}\left(1-\alpha_{3}^{j}\right) \alpha_{2}^{3} \alpha_{3}^{3}\left\|U_{2} x_{0}-x_{0}\right\|^{2} \\
& +\left(1-\prod_{j=3}^{N}\left(1-\alpha_{3}^{j}\right)\right)\left\|x_{0}-x^{*}\right\|^{2} \\
\leq & \prod_{j=3}^{N}\left(1-\alpha_{3}^{j}\right)\left\|S_{2} x_{0}-x^{*}\right\|^{2} \\
& -\prod_{j=4}^{N}\left(1-\alpha_{3}^{j}\right) \alpha_{2}^{3} \alpha_{3}^{3}\left\|U_{2} x_{0}-x_{0}\right\|^{2} \\
& +\left(1-\prod_{j=3}^{N}\left(1-\alpha_{3}^{j}\right)\right)\left\|x_{0}-x^{*}\right\|^{2} \\
\leq & \left\|x_{0}-x^{*}\right\|^{2}-\prod_{j=4}^{N}\left(1-\alpha_{3}^{j}\right) \alpha_{2}^{3} \alpha_{3}^{3}\left\|U_{2} x_{0}-x_{0}\right\|^{2} .
\end{aligned}
$$

It follows that

$$
\prod_{j=4}^{N}\left(1-\alpha_{3}^{j}\right) \alpha_{2}^{3} \alpha_{3}^{3}\left\|U_{2} x_{0}-x_{0}\right\|^{2} \leq 0 .
$$

It implies that

$$
x_{0}=U_{2} x_{0} .
$$

By (2.9) and (2.10), we have $x_{0} \in F\left(S_{2}\right)$. Hence, we have

$$
x_{0} \in F\left(S_{2}\right) \cap F\left(T_{2}\right) .
$$

By continuing in this way, we can show that $x_{0} \in F\left(S_{i}\right) \cap F\left(T_{i}\right)$ and $x_{0}=U_{i} x_{0}$ for all $i=$ $1,2, \ldots, N-1$. Finally, we shall show that $x_{0} \in F\left(S_{N}\right) \cap F\left(T_{N}\right)$. Since

$$
\begin{aligned}
\left\|S^{A} x_{0}-x^{*}\right\|^{2} \leq & \left(1-\alpha_{3}^{N}\right)\left\|U_{N-1} x_{0}-x^{*}\right\|^{2} \\
& +\alpha_{1}^{N}\left(\kappa-\alpha_{2}^{N}\right)\left\|\left(I-T_{N}\right) U_{N-1} x_{0}\right\|^{2} \\
& +\left(1-\left(1-\alpha_{3}^{N}\right)\right)\left\|x_{0}-x^{*}\right\|^{2} \\
= & \left(1-\alpha_{3}^{N}\right)\left\|S_{N-1} x_{0}-x^{*}\right\|^{2}+\alpha_{1}^{N}\left(\kappa-\alpha_{2}^{N}\right)\left\|\left(I-T_{N}\right) x_{0}\right\|^{2} \\
& +\left(1-\left(1-\alpha_{3}^{N}\right)\right)\left\|x_{0}-x^{*}\right\|^{2} \\
\leq & \left\|x_{0}-x^{*}\right\|^{2}+\alpha_{1}^{N}\left(\kappa-\alpha_{2}^{N}\right)\left\|\left(I-T_{N}\right) x_{0}\right\|^{2} .
\end{aligned}
$$

It implies that

$$
\alpha_{1}^{N}\left(\alpha_{2}^{N}-\kappa\right)\left\|\left(I-T_{N}\right) x_{0}\right\|^{2} \leq 0,
$$


which implies that $x_{0}=T_{N} x_{0}$, that is, $x_{0} \in F\left(T_{N}\right)$. It implies that

$$
x_{0}=S^{A} x_{0}=S_{N}\left(\alpha_{1}^{N} T_{N} U_{N-1} x_{0}+\alpha_{2}^{N} U_{N-1} x_{0}+\alpha_{3}^{N} x_{0}\right)=S_{N} x_{0} .
$$

Then we have $x_{0} \in F\left(S_{N}\right) \cap F\left(T_{N}\right)$. Hence $F\left(S^{A}\right) \subseteq \bigcap_{i=1}^{N} F\left(S_{i}\right) \cap \bigcap_{i=1}^{N} F\left(T_{i}\right)$.

Applying (2.4), we have that the mapping $S^{A}$ is a nonexpansive.

Lemma 2.9 Let $C$ be a nonempty closed convex subset of a real Hilbert space. Let $\left\{T_{i}\right\}_{i=1}^{N}$ be a finite family of $\kappa_{i}$-strict pseudo-contractions of $C$ into itself, and let $\left\{S_{i}\right\}_{i=1}^{N}$ be a finite family of nonexpansive mappings of $C$ into itself with $\kappa=\max \left\{\kappa_{i}: i=1,2, \ldots, N\right\}$, and let $\alpha_{j}^{(n)}=\left(\alpha_{1}^{n, j}, \alpha_{2}^{n, j}, \alpha_{3}^{n, j}\right), \alpha_{j}=\left(\alpha_{1}^{j}, \alpha_{2}^{j}, \alpha_{3}^{j}\right) \in I \times I \times I$, where $I=[0,1], \alpha_{1}^{n, j}+\alpha_{2}^{n, j}+\alpha_{3}^{n, j}=1$ and $\alpha_{1}^{j}+$ $\alpha_{2}^{j}+\alpha_{3}^{j}=1$ such that $\alpha_{i}^{n, j} \rightarrow \alpha_{i}^{j} \in[0,1]$ as $n \rightarrow \infty$ for $i=1,3$ and $j=1,2,3, \ldots, N$. Moreover, for every $n \in \mathbb{N}$, let $S^{A}$ and $S_{n}^{A}$ be the $S^{A}$-mapping generated by $S_{1}, S_{2}, \ldots, S_{N}, T_{1}, T_{2}, \ldots, T_{N}$ and $\alpha_{1}, \alpha_{2}, \ldots, \alpha_{N}$ and $S_{1}, S_{2}, \ldots, S_{N}, T_{1}, T_{2}, \ldots, T_{N}$ and $\alpha_{1}^{(n)}, \alpha_{2}^{(n)}, \ldots, \alpha_{N}^{(n)}$, respectively. Then $\lim _{n \rightarrow \infty}\left\|S_{n}^{A} x_{n}-S^{A} x_{n}\right\|=0$ for every bounded sequence $\left\{x_{n}\right\}$ in $C$.

Proof Let $\left\{x_{n}\right\}$ be a bounded sequence in $C, U_{k}$ and $U_{n, k}$ be generated by $S_{1}, S_{2}, \ldots, S_{N}$, $T_{1}, T_{2}, \ldots, T_{N}$ and $\alpha_{1}, \alpha_{2}, \ldots, \alpha_{N}$ and $S_{1}, S_{2}, \ldots, S_{N}, T_{1}, T_{2}, \ldots, T_{N}$ and $\alpha_{1}^{(n)}, \alpha_{2}^{(n)}, \ldots, \alpha_{N}^{(n)}$, respectively. For each $n \in \mathbb{N}$, we have

$$
\begin{aligned}
\left\|U_{n, 1} x_{n}-U_{1} x_{n}\right\| & =\left\|S_{1}\left(\alpha_{1}^{n, 1} T_{1} x_{n}+\left(1-\alpha_{1}^{n, 1}\right) x_{n}\right)-S_{1}\left(\alpha_{1}^{1} T_{1} x_{n}+\left(1-\alpha_{1}^{1}\right) x_{n}\right)\right\| \\
& \leq\left\|\alpha_{1}^{n, 1} T_{1} x_{n}+\left(1-\alpha_{1}^{n, 1}\right) x_{n}-\alpha_{1}^{1} T_{1} x_{n}-\left(1-\alpha_{1}^{1}\right) x_{n}\right\| \\
& =\left|\alpha_{1}^{n, 1}-\alpha_{1}^{1}\right|\left\|T_{1} x_{n}-x_{n}\right\|,
\end{aligned}
$$

and for $k \in\{2,3, \ldots, N\}$, by using Lemma 2.4 , we obtain

$$
\begin{aligned}
\left\|U_{n, k} x_{n}-U_{k} x_{n}\right\|= & \| S_{k}\left(\alpha_{1}^{n, k} T_{k} U_{n, k-1} x_{n}+\alpha_{2}^{n, k} U_{n, k-1} x_{n}+\alpha_{3}^{n, k} x_{n}\right) \\
& -S_{k}\left(\alpha_{1}^{k} T_{k} U_{k-1} x_{n}+\alpha_{2}^{k} U_{k-1} x_{n}+\alpha_{3}^{k} x_{n}\right) \| \\
\leq & \| \alpha_{1}^{n, k} T_{k} U_{n, k-1} x_{n}+\alpha_{2}^{n, k} U_{n, k-1} x_{n}+\alpha_{3}^{n, k} x_{n} \\
& -\alpha_{1}^{k} T_{k} U_{k-1} x_{n}-\alpha_{2}^{k} U_{k-1} x_{n}-\alpha_{3}^{k} x_{n} \| \\
= & \| \alpha_{1}^{n, k}\left(T_{k} U_{n, k-1} x_{n}-T_{k} U_{k-1} x_{n}\right)+\left(\alpha_{1}^{n, k}-\alpha_{1}^{k}\right) T_{k} U_{k-1} x_{n} \\
& +\left(\alpha_{3}^{n, k}-\alpha_{3}^{k}\right) x_{n}+\alpha_{2}^{n, k}\left(U_{n, k-1} x_{n}-U_{k-1} x_{n}\right) \\
& +\left(\alpha_{2}^{n, k}-\alpha_{2}^{k}\right) U_{k-1} x_{n} \| \\
\leq & \alpha_{1}^{n, k}\left\|T_{k} U_{n, k-1} x_{n}-T_{k} U_{k-1} x_{n}\right\|+\left|\alpha_{1}^{n, k}-\alpha_{1}^{k}\right|\left\|T_{k} U_{k-1} x_{n}\right\| \\
& +\left|\alpha_{3}^{n, k}-\alpha_{3}^{k}\right|\left\|x_{n}\right\|+\alpha_{2}^{n, k}\left\|U_{n, k-1} x_{n}-U_{k-1} x_{n}\right\| \\
& +\left|\alpha_{2}^{n, k}-\alpha_{2}^{k}\right|\left\|U_{k-1} x_{n}\right\| \\
= & \alpha_{1}^{n, k}\left\|T_{k} U_{n, k-1} x_{n}-T_{k} U_{k-1} x_{n}\right\|+\left|\alpha_{1}^{n, k}-\alpha_{1}^{k}\right|\left\|T_{k} U_{k-1} x_{n}\right\| \\
& +\alpha_{2}^{n, k}\left\|U_{n, k-1} x_{n}-U_{k-1} x_{n}\right\|+\mid 1-\alpha_{1}^{n, k}-\alpha_{3}^{n, k}-1 \\
& +\alpha_{1}^{k}+\alpha_{3}^{k}\left|\left\|U_{k-1} x_{n}\right\|+\right| \alpha_{3}^{n, k}-\alpha_{3}^{k} \mid\left\|x_{n}\right\| \\
\leq & \alpha_{1}^{n, k} \frac{1+\kappa}{1-\kappa}\left\|U_{n, k-1} x_{n}-U_{k-1} x_{n}\right\|
\end{aligned}
$$




$$
\begin{aligned}
& +\left|\alpha_{1}^{n, k}-\alpha_{1}^{k}\right|\left\|T_{k} U_{k-1} x_{n}\right\|+\alpha_{2}^{n, k}\left\|U_{n, k-1} x_{n}-U_{k-1} x_{n}\right\| \\
& +\left(\left|\alpha_{1}^{k}-\alpha_{1}^{n, k}\right|+\left|\alpha_{3}^{n, k}-\alpha_{3}^{k}\right|\right)\left\|U_{k-1} x_{n}\right\|+\left|\alpha_{3}^{n, k}-\alpha_{3}^{k}\right|\left\|x_{n}\right\| \\
\leq & \frac{1+\kappa}{1-\kappa}\left\|U_{n, k-1} x_{n}-U_{k-1} x_{n}\right\|+\left|\alpha_{1}^{n, k}-\alpha_{1}^{k}\right|\left\|T_{k} U_{k-1} x_{n}\right\| \\
& +\frac{1-\kappa}{1-\kappa}\left\|U_{n, k-1} x_{n}-U_{k-1} x_{n}\right\|+\left(\left|\alpha_{1}^{k}-\alpha_{1}^{n, k}\right|\right. \\
& \left.+\left|\alpha_{3}^{n, k}-\alpha_{3}^{k}\right|\right)\left\|U_{k-1} x_{n}\right\|+\left|\alpha_{3}^{n, k}-\alpha_{3}^{k}\right|\left\|x_{n}\right\| \\
\leq & \frac{2}{1-\kappa}\left\|U_{n, k-1} x_{n}-U_{k-1} x_{n}\right\|+\left|\alpha_{1}^{n, k}-\alpha_{1}^{k}\right|\left(\left\|T_{k} U_{k-1} x_{n}\right\|+\left\|U_{k-1} x_{n}\right\|\right) \\
& +\left|\alpha_{3}^{n, k}-\alpha_{3}^{k}\right|\left(\left\|U_{k-1} x_{n}\right\|+\left\|x_{n}\right\|\right) .
\end{aligned}
$$

By (2.13) and (2.14), we have

$$
\begin{aligned}
& \left\|S_{n}^{A} x_{n}-S^{A} x_{n}\right\|=\left\|U_{n, N} x_{n}-U_{N} x_{n}\right\| \\
& \leq \frac{2}{1-\kappa}\left\|U_{n, N-1} x_{n}-U_{N-1} x_{n}\right\|+\left|\alpha_{1}^{n, N}-\alpha_{1}^{N}\right|\left(\left\|T_{N} U_{N-1} x_{n}\right\|\right. \\
& \left.+\left\|U_{N-1} x_{n}\right\|\right)+\left|\alpha_{3}^{n, N}-\alpha_{3}^{N}\right|\left(\left\|U_{N-1} x_{n}\right\|+\left\|x_{n}\right\|\right) \\
& \leq \frac{2}{1-\kappa}\left(\frac{2}{1-\kappa}\left\|U_{n, N-2} x_{n}-U_{N-2} x_{n}\right\|\right. \\
& +\left|\alpha_{1}^{n, N-1}-\alpha_{1}^{N-1}\right|\left(\left\|T_{N-1} U_{N-2} x_{n}\right\|+\left\|U_{N-2} x_{n}\right\|\right) \\
& \left.+\left|\alpha_{3}^{n, N-1}-\alpha_{3}^{N-1}\right|\left(\left\|U_{N-2} x_{n}\right\|+\left\|x_{n}\right\|\right)\right) \\
& +\left|\alpha_{1}^{n, N}-\alpha_{1}^{N}\right|\left(\left\|T_{N} U_{N-1} x_{n}\right\|+\left\|U_{N-1} x_{n}\right\|\right) \\
& +\left|\alpha_{3}^{n, N}-\alpha_{3}^{N}\right|\left(\left\|U_{N-1} x_{n}\right\|+\left\|x_{n}\right\|\right) \\
& =\left(\frac{2}{1-\kappa}\right)^{2}\left\|U_{n, N-2} x_{n}-U_{N-2} x_{n}\right\| \\
& +\sum_{j=N-1}^{N}\left(\frac{2}{1-\kappa}\right)^{N-j}\left|\alpha_{1}^{n, j}-\alpha_{1}^{j}\right|\left(\left\|T_{j} U_{j-1} x_{n}\right\|+\left\|U_{j-1} x_{n}\right\|\right) \\
& +\sum_{j=N-1}^{N}\left(\frac{2}{1-\kappa}\right)^{N-j}\left|\alpha_{3}^{n, j}-\alpha_{3}^{j}\right|\left(\left\|U_{j-1} x_{n}\right\|+\left\|x_{n}\right\|\right) \\
& \leq \cdots \\
& \leq\left(\frac{2}{1-\kappa}\right)^{N-1}\left\|U_{n, 1} x_{n}-U_{1} x_{n}\right\| \\
& +\sum_{j=2}^{N}\left(\frac{2}{1-\kappa}\right)^{N-j}\left|\alpha_{1}^{n, j}-\alpha_{1}^{j}\right|\left(\left\|T_{j} U_{j-1} x_{n}\right\|+\left\|U_{j-1} x_{n}\right\|\right) \\
& +\sum_{j=2}^{N}\left(\frac{2}{1-\kappa}\right)^{N-j}\left|\alpha_{3}^{n, j}-\alpha_{3}^{j}\right|\left(\left\|U_{j-1} x_{n}\right\|+\left\|x_{n}\right\|\right) \\
& =\left(\frac{2}{1-\kappa}\right)^{N-1}\left|\alpha_{1}^{n, 1}-\alpha_{1}^{1}\right|\left\|T_{1} x_{n}-x_{n}\right\|
\end{aligned}
$$




$$
\begin{aligned}
& +\sum_{j=2}^{N}\left(\frac{2}{1-\kappa}\right)^{N-j}\left|\alpha_{1}^{n, j}-\alpha_{1}^{j}\right|\left(\left\|T_{j} U_{j-1} x_{n}\right\|+\left\|U_{j-1} x_{n}\right\|\right) \\
& +\sum_{j=2}^{N}\left(\frac{2}{1-\kappa}\right)^{N-j}\left|\alpha_{3}^{n, j}-\alpha_{3}^{j}\right|\left(\left\|U_{j-1} x_{n}\right\|+\left\|x_{n}\right\|\right)
\end{aligned}
$$

This together with the assumption $\alpha_{i}^{n, j} \rightarrow \alpha_{i}^{j}$ as $n \rightarrow \infty(i=1,3, j=1,2, \ldots, N)$, we can conclude that

$$
\lim _{n \rightarrow \infty}\left\|S_{n}^{A} x_{n}-S^{A} x_{n}\right\|=0
$$

Lemma 2.10 Let $C$ be a nonempty closed convex subset of a real Hilbert space. Let $\left\{T_{i}\right\}_{i=1}^{N}$ be a finite family of $\kappa_{i}$-strict pseudo-contractions of $C$ into itself, and let $\left\{S_{i}\right\}_{i=1}^{N}$ be a finite family of nonexpansive mappings of $C$ into itself with $\kappa=\max \left\{\kappa_{i}: i=1,2, \ldots, N\right\}$, and let $\alpha_{j}^{(n)}=\left(\alpha_{1}^{n, j}, \alpha_{2}^{n, j}, \alpha_{3}^{n, j}\right), \alpha_{j}=\left(\alpha_{1}^{j}, \alpha_{2}^{j}, \alpha_{3}^{j}\right) \in I \times I \times I$, where $I=[0,1], \alpha_{1}^{n, j}+\alpha_{2}^{n, j}+\alpha_{3}^{n, j}=1$ and $\alpha_{1}^{j}+$ $\alpha_{2}^{j}+\alpha_{3}^{j}=1$ such that $\sum_{n=1}^{\infty}\left|\alpha_{1}^{n+1, j}-\alpha_{1}^{n, j}\right|<\infty, \sum_{n=1}^{\infty}\left|\alpha_{3}^{n+1, j}-\alpha_{3}^{n, j}\right|<\infty$ for all $j \in\{1,2,3, \ldots, N\}$. For every $n \in \mathbb{N}$, let $S_{n}^{A}$ be the $S^{A}$-mapping generated by $S_{1}, S_{2}, \ldots, S_{N}, T_{1}, T_{2}, \ldots, T_{N}$ and $\alpha_{1}^{(n)}, \alpha_{2}^{(n)}, \ldots, \alpha_{N}^{(n)}$. Then $\sum_{n=1}^{\infty}\left\|S_{n+1}^{A} z_{n}-S_{n}^{A} z_{n}\right\|<\infty$ for every bounded sequence $\left\{z_{n}\right\}$ in $C$.

Proof Let $\left\{z_{n}\right\}$ be a bounded sequence in $C$. For each $n \in \mathbb{N}$ and the definition of $S^{A}$, we have

$$
\begin{aligned}
\left\|U_{n+1,1} z_{n}-U_{n, 1} z_{n}\right\| & =\left\|S_{1}\left(\alpha_{1}^{n+1,1} T_{1} z_{n}+\left(1-\alpha_{1}^{n+1,1}\right) z_{n}\right)-S_{1}\left(\alpha_{1}^{n, 1} T_{1} z_{n}+\left(1-\alpha_{1}^{n, 1}\right) z_{n}\right)\right\| \\
& \leq\left\|\alpha_{1}^{n+1,1} T_{1} z_{n}+\left(1-\alpha_{1}^{n+1,1}\right) z_{n}-\alpha_{1}^{n, 1} T_{1} z_{n}-\left(1-\alpha_{1}^{n, 1}\right) z_{n}\right\| \\
& =\left|\alpha_{1}^{n+1,1}-\alpha_{1}^{n, 1}\right|\left\|T_{1} z_{n}-z_{n}\right\| .
\end{aligned}
$$

For $k \in\{2,3, \ldots, N\}$, and using the same method as (2.14) in Lemma 2.9, we have

$$
\begin{aligned}
\left\|U_{n+1, k} z_{n}-U_{n, k} z_{n}\right\| \leq & \frac{2}{1-\kappa}\left\|U_{n+1, k-1} z_{n}-U_{n, k-1} z_{n}\right\|+\left|\alpha_{1}^{n+1, k}-\alpha_{1}^{n, k}\right|\left(\left\|T_{k} U_{n, k-1} z_{n}\right\|\right. \\
& \left.+\left\|U_{n, k-1} z_{n}\right\|\right)+\left|\alpha_{3}^{n+1, k}-\alpha_{3}^{n, k}\right|\left(\left\|U_{n, k-1} z_{n}\right\|+\left\|z_{n}\right\|\right) .
\end{aligned}
$$

From (2.16), (2.17), and using the same method as (2.15) in Lemma 2.9, we have

$$
\begin{aligned}
\left\|S_{n+1}^{A} z_{n}-S_{n}^{A} z_{n}\right\| \leq & \left(\frac{2}{1-\kappa}\right)^{N-1}\left|\alpha_{1}^{n+1,1}-\alpha_{1}^{n, 1}\right|\left\|T_{1} z_{n}-z_{n}\right\| \\
& +\sum_{j=2}^{N}\left(\frac{2}{1-\kappa}\right)^{N-j}\left|\alpha_{1}^{n+1, j}-\alpha_{1}^{n, j}\right|\left(\left\|T_{j} U_{n, j-1} z_{n}\right\|+\left\|U_{n, j-1} z_{n}\right\|\right) \\
& +\sum_{j=2}^{N}\left(\frac{2}{1-\kappa}\right)^{N-j}\left|\alpha_{3}^{n+1, j}-\alpha_{3}^{n, j}\right|\left(\left\|U_{n, j-1} z_{n}\right\|+\left\|z_{n}\right\|\right) .
\end{aligned}
$$

It implies that

$$
\sum_{n=1}^{\infty}\left\|S_{n+1}^{A} z_{n}-S_{n}^{A} z_{n}\right\|<\infty
$$




\section{Main result}

Theorem 3.1 Let $C$ be a nonempty closed convex subset of Hilbert spaces $H$, and let $f$ be an $\alpha$-contraction on $H$. Let $F_{i}$ be a bifunction from $C \times C$ into $\mathbb{R}$, for every $i=1,2, \ldots, N$ satisfying (A1)-(A4). Let $\left\{T_{i}\right\}_{i=1}^{N}$ be a finite family of $\kappa_{i}$-strict pseudo-contractions of $C$ into itself, and let $\left\{S_{i}\right\}_{i=1}^{N}$ be a finite family of nonexpansive mappings of $C$ into itself with $\mathbb{F} \equiv$ $\bigcap_{i=1}^{N} F\left(S_{i}\right) \cap \bigcap_{i=1}^{N} F\left(T_{i}\right) \cap \bigcap_{i=1}^{N} E P\left(F_{i}\right) \neq \emptyset$ and $\kappa=\max \left\{\kappa_{i}: i=1,2, \ldots, N\right\}$, and let $\alpha_{j}^{(n)}=$ $\left(\alpha_{1}^{n, j}, \alpha_{2}^{n, j}, \alpha_{3}^{n, j}\right) \in I \times I \times I, j=1,2,3, \ldots, N$, where $I=[0,1], \alpha_{1}^{n, j}+\alpha_{2}^{n, j}+\alpha_{3}^{n, j}=1, \alpha_{1}^{n, j}, \alpha_{2}^{n, j}, \alpha_{3}^{n, j} \in$ $[a, b] \subset(\kappa, 1)$ for all $j=1,2, \ldots, N$. Let $S_{n}^{A}$ be the $S^{A}$-mapping generated by $S_{1}, S_{2}, \ldots, S_{N}$, $T_{1}, T_{2}, \ldots, T_{N}$ and $\alpha_{1}^{(n)}, \alpha_{2}^{(n)}, \ldots, \alpha_{N}^{(n)}$. Let $\left\{x_{n}\right\}$ and $\left\{z_{n}\right\}$ be the sequences generated by $x_{1} \in C$ and

$$
\left\{\begin{array}{l}
F_{i}\left(u_{n}^{i}, y\right)+\frac{1}{r_{n}^{i}}\left\langle y-u_{n}^{i}, u_{n}^{i}-x_{n}\right\rangle \geq 0, \quad \forall y \in C \text { and } i=1,2, \ldots, N, \\
z_{n}=\sum_{i=1}^{N} \delta_{n}^{i} u_{n}^{i}, \\
x_{n+1}=\alpha_{n} f\left(z_{n}\right)+\left(1-\alpha_{n}\right) S_{n}^{A} z_{n}, \quad \forall n \geq 1,
\end{array}\right.
$$

where $\left\{\alpha_{n}\right\}$ is a sequence in $[0,1]$. Assume that the following conditions hold:

(i) $\lim _{n \rightarrow \infty} \alpha_{n}=0, \sum_{n=1}^{\infty} \alpha_{n}=\infty$;

(ii) $\sum_{n=1}^{\infty}\left|\alpha_{1}^{n+1, j}-\alpha_{1}^{n, j}\right|<\infty, \sum_{n=1}^{\infty}\left|\alpha_{3}^{n+1, j}-\alpha_{3}^{n, j}\right|<\infty$, for all $j \in\{1,2,3, \ldots, N\}$ and $\sum_{n=1}^{\infty}\left|\alpha_{n+1}-\alpha_{n}\right|<\infty$;

(iii) $\sum_{i=1}^{N} \delta_{n}^{i}=1, \sum_{n=1}^{\infty}\left|\delta_{n+1}^{i}-\delta_{n}^{i}\right|<\infty$ and $\lim _{n \rightarrow \infty} \delta_{n}^{i}=\delta_{i} \in(\kappa, 1)$, for every $i=1,2, \ldots, N$;

(iv) $\kappa<\theta \leq r_{n}^{i} \leq \eta$, for every $i=1,2, \ldots, N$ and $\sum_{n=1}^{\infty}\left|r_{n+1}^{i}-r_{n}^{i}\right|<\infty$.

Then the sequence $\left\{x_{n}\right\}$ converges strongly to $x^{*}=P_{\mathbb{F}} f\left(x^{*}\right)$.

Proof Let $p \in \mathbb{F}$, we have $p \in \bigcap_{i=1}^{N} E P\left(F_{i}\right)$ from Lemma 2.6, we obtain $p \in \bigcap_{i=1}^{N} F\left(T_{r_{n}^{i}}\right)$. Since

$$
F_{i}\left(u_{n}^{i}, y\right)+\frac{1}{r_{n}^{i}}\left\langle y-u_{n}^{i}, u_{n}^{i}-x_{n}\right) \geq 0, \quad \forall y \in C \text { and } i=1,2, \ldots, N
$$

Again from Lemma 2.6, we have $u_{n}^{i}=T_{r_{n}^{i}} x_{n}$ for every $i=1,2, \ldots, N$. By definition of $x_{n}$, we have

$$
\begin{aligned}
\left\|x_{n+1}-p\right\| & \leq \alpha_{n}\left\|f\left(z_{n}\right)-p\right\|+\left(1-\alpha_{n}\right)\left\|S_{n}^{A} z_{n}-p\right\| \\
& \leq \alpha_{n}\left\|f\left(z_{n}\right)-f(p)\right\|+\alpha_{n}\|f(p)-p\|+\left(1-\alpha_{n}\right)\left\|S_{n}^{A} z_{n}-p\right\| \\
& \leq \alpha_{n} \alpha\left\|z_{n}-p\right\|+\alpha_{n}\|f(p)-p\|+\left(1-\alpha_{n}\right)\left\|z_{n}-p\right\| \\
& =\alpha_{n}\|f(p)-p\|+\left(1-\alpha_{n}(1-\alpha)\right)\left\|z_{n}-p\right\| \\
& =\alpha_{n}\|f(p)-p\|+\left(1-\alpha_{n}(1-\alpha)\right)\left\|\sum_{i=1}^{N} \delta_{n}^{i}\left(u_{n}^{i}-p\right)\right\| \\
& \leq \alpha_{n}\|f(p)-p\|+\left(1-\alpha_{n}(1-\alpha)\right) \sum_{i=1}^{N} \delta_{n}^{i}\left\|u_{n}^{i}-p\right\| \\
& \leq \alpha_{n}\|f(p)-p\|+\left(1-\alpha_{n}(1-\alpha)\right)\left\|x_{n}-p\right\| .
\end{aligned}
$$

Put $K=\max \left\{\left\|x_{1}-p\right\|, \frac{\|f(p)-p\|}{1-\alpha}\right\}$. By (3.3), we can show by induction that $\left\|x_{n}-p\right\| \leq K$, $\forall n \in \mathbb{N}$. This implies that $\left\{x_{n}\right\}$ is bounded, and so are $\left\{u_{n}^{i}\right\}$, for every $i=1,2, \ldots, N$ and $\left\{z_{n}\right\}$. 
Next, we will show that

$$
\lim _{n \rightarrow \infty}\left\|x_{n+1}-x_{n}\right\|=0
$$

By nonexpansiveness of $x_{n}$, we have

$$
\begin{aligned}
& \left\|x_{n+1}-x_{n}\right\|=\left\|\alpha_{n} f\left(z_{n}\right)+\left(1-\alpha_{n}\right) S_{n}^{A} z_{n}-\alpha_{n-1} f\left(z_{n-1}\right)-\left(1-\alpha_{n-1}\right) S_{n-1}^{A} z_{n-1}\right\| \\
& =\| \alpha_{n}\left(f\left(z_{n}\right)-f\left(z_{n-1}\right)\right)+\left(\alpha_{n}-\alpha_{n-1}\right) f\left(z_{n-1}\right)+\left(1-\alpha_{n}\right)\left(S_{n}^{A} z_{n}-S_{n-1}^{A} z_{n-1}\right) \\
& +\left(\alpha_{n-1}-\alpha_{n}\right) S_{n-1}^{A} z_{n-1} \| \\
& \leq \alpha_{n}\left\|f\left(z_{n}\right)-f\left(z_{n-1}\right)\right\|+\left|\alpha_{n}-\alpha_{n-1}\right|\left\|f\left(z_{n-1}\right)\right\|+\left(1-\alpha_{n}\right)\left\|S_{n}^{A} z_{n}-S_{n-1}^{A} z_{n-1}\right\| \\
& +\left|\alpha_{n-1}-\alpha_{n}\right|\left\|S_{n-1}^{A} z_{n-1}\right\| \\
& \leq \alpha_{n} \alpha\left\|z_{n}-z_{n-1}\right\|+\left|\alpha_{n}-\alpha_{n-1}\right|\left\|f\left(z_{n-1}\right)\right\| \\
& +\left(1-\alpha_{n}\right)\left(\left\|S_{n}^{A} z_{n}-S_{n}^{A} z_{n-1}\right\|+\left\|S_{n}^{A} z_{n-1}-S_{n-1}^{A} z_{n-1}\right\|\right) \\
& +\left|\alpha_{n-1}-\alpha_{n}\right|\left\|S_{n-1}^{A} z_{n-1}\right\| \\
& \leq\left(1-\alpha_{n}(1-\alpha)\right)\left\|z_{n}-z_{n-1}\right\|+\left|\alpha_{n}-\alpha_{n-1}\right|\left\|f\left(z_{n-1}\right)\right\| \\
& +\left(1-\alpha_{n}\right)\left\|S_{n}^{A} z_{n-1}-S_{n-1}^{A} z_{n-1}\right\|+\left|\alpha_{n-1}-\alpha_{n}\right|\left\|S_{n-1}^{A} z_{n-1}\right\| \\
& =\left(1-\alpha_{n}(1-\alpha)\right)\left(\left\|\sum_{i=1}^{N} \delta_{n}^{i} u_{n}^{i}-\sum_{i=1}^{N} \delta_{n-1}^{i} u_{n-1}^{i}\right\|\right)+\left|\alpha_{n}-\alpha_{n-1}\right|\left\|f\left(z_{n-1}\right)\right\| \\
& +\left(1-\alpha_{n}\right)\left\|S_{n}^{A} z_{n-1}-S_{n-1}^{A} z_{n-1}\right\|+\left|\alpha_{n-1}-\alpha_{n}\right|\left\|S_{n-1}^{A} z_{n-1}\right\| \\
& =\left(1-\alpha_{n}(1-\alpha)\right)\left(\left\|\sum_{i=1}^{N} \delta_{n}^{i}\left(u_{n}^{i}-u_{n-1}^{i}\right)+\sum_{i=1}^{N}\left(\delta_{n}^{i}-\delta_{n-1}^{i}\right) u_{n-1}^{i}\right\|\right) \\
& +\left|\alpha_{n}-\alpha_{n-1}\right|\left\|f\left(z_{n-1}\right)\right\|+\left(1-\alpha_{n}\right)\left\|S_{n}^{A} z_{n-1}-S_{n-1}^{A} z_{n-1}\right\| \\
& +\left|\alpha_{n-1}-\alpha_{n}\right|\left\|S_{n-1}^{A} z_{n-1}\right\| \\
& \leq\left(1-\alpha_{n}(1-\alpha)\right)\left(\sum_{i=1}^{N} \delta_{n}^{i}\left\|u_{n}^{i}-u_{n-1}^{i}\right\|+\sum_{i=1}^{N}\left|\delta_{n}^{i}-\delta_{n-1}^{i}\right|\left\|u_{n-1}^{i}\right\|\right) \\
& +\left|\alpha_{n}-\alpha_{n-1}\right|\left\|f\left(z_{n-1}\right)\right\|+\left(1-\alpha_{n}\right)\left\|S_{n}^{A} z_{n-1}-S_{n-1}^{A} z_{n-1}\right\| \\
& +\left|\alpha_{n-1}-\alpha_{n}\right|\left\|S_{n-1}^{A} z_{n-1}\right\| \text {. }
\end{aligned}
$$

From Lemma 2.10, we have

$$
\sum_{n=1}^{\infty}\left\|S_{n+1}^{A} z_{n}-S_{n}^{A} z_{n}\right\|<\infty
$$

Since $u_{n}^{i}=T_{r_{n}^{i}} x_{n}$ for every $i=1,2, \ldots, N$. By definition of $T_{r_{n}^{i}}$, we have

$$
F\left(T_{r_{n}^{i}} x_{n}, y\right)+\frac{1}{r_{n}^{i}}\left\langle y-T_{r_{n}^{i}} x_{n}, T_{r_{n}^{i}} x_{n}-x_{n}\right\rangle \geq 0, \quad \forall y \in C,
$$

similarly,

$$
F\left(T_{r_{n+1}^{i}} x_{n+1}, y\right)+\frac{1}{r_{n+1}^{i}}\left\langle y-T_{r_{n+1}^{i}} x_{n+1}, T_{r_{n+1}^{i}} x_{n+1}-x_{n+1}\right\rangle \geq 0, \quad \forall y \in C .
$$


From (3.7) and (3.8), we obtain

$$
F\left(T_{r_{n}^{i}} x_{n}, T_{r_{n+1}^{i}} x_{n+1}\right)+\frac{1}{r_{n}^{i}}\left\langle T_{r_{n+1}^{i}} x_{n+1}-T_{r_{n}^{i}} x_{n}, T_{r_{n}^{i}} x_{n}-x_{n}\right\rangle \geq 0
$$

and

$$
F\left(T_{r_{n+1}^{i}} x_{n+1}, T_{r_{n}^{i}} x_{n}\right)+\frac{1}{r_{n+1}^{i}}\left\langle T_{r_{n}^{i}} x_{n}-T_{r_{n+1}^{i}} x_{n+1}, T_{r_{n+1}^{i}} x_{n+1}-x_{n+1}\right\rangle \geq 0
$$

By (3.9) and (3.10), we have

$$
\frac{1}{r_{n}^{i}}\left\langle T_{r_{n+1}^{i}} x_{n+1}-T_{r_{n}^{i}} x_{n}, T_{r_{n}^{i}} x_{n}-x_{n}\right\rangle+\frac{1}{r_{n+1}^{i}}\left\langle T_{r_{n}^{i}} x_{n}-T_{r_{n+1}^{i}} x_{n+1}, T_{r_{n+1}^{i}} x_{n+1}-x_{n+1}\right\rangle \geq 0 .
$$

It follows that

$$
\left\langle T_{r_{n}^{i}} x_{n}-T_{r_{n+1}^{i}} x_{n+1}, \frac{T_{r_{n+1}^{i}} x_{n+1}-x_{n+1}}{r_{n+1}^{i}}-\frac{T_{r_{n}^{i}} x_{n}-x_{n}}{r_{n}^{i}}\right\rangle \geq 0
$$

This implies that

$$
0 \leq\left\langle T_{r_{n+1}^{i}} x_{n+1}-T_{r_{n}^{i}} x_{n}, T_{r_{n}^{i}} x_{n}-T_{r_{n+1}^{i}} x_{n+1}+T_{r_{n+1}^{i}} x_{n+1}-x_{n}-\frac{r_{n}^{i}}{r_{n+1}^{i}}\left(T_{r_{n+1}^{i}} x_{n+1}-x_{n+1}\right)\right\rangle .
$$

It follows that

$$
\begin{aligned}
& \left\|T_{r_{n+1}^{i}} x_{n+1}-T_{r_{n}^{i}} x_{n}\right\|^{2} \\
& \quad \leq\left\langle T_{r_{n+1}^{i}} x_{n+1}-T_{r_{n}^{i}} x_{n}, T_{r_{n+1}^{i}} x_{n+1}-x_{n}-\frac{r_{n}^{i}}{r_{n+1}^{i}}\left(T_{r_{n+1}^{i}} x_{n+1}-x_{n+1}\right)\right\rangle \\
& \quad=\left\langle T_{r_{n+1}^{i} x_{n+1}}-T_{r_{n}^{i}} x_{n}, x_{n+1}-x_{n}+\left(1-\frac{r_{n}^{i}}{r_{n+1}^{i}}\right)\left(T_{r_{n+1}^{i}} x_{n+1}-x_{n+1}\right)\right\rangle \\
& \leq\left\|T_{r_{n+1}^{i}} x_{n+1}-T_{r_{n}^{i}} x_{n}\right\|\left\|x_{n+1}-x_{n}+\left(1-\frac{r_{n}^{i}}{r_{n+1}^{i}}\right)\left(T_{r_{n+1}^{i}} x_{n+1}-x_{n+1}\right)\right\| \\
& \quad \leq\left\|T_{r_{n+1}^{i}} x_{n+1}-T_{r_{n}^{i}} x_{n}\right\|\left(\left\|x_{n+1}-x_{n}\right\|+\left|1-\frac{r_{n}^{i}}{r_{n+1}^{i}}\right|\left\|T_{r_{n+1}^{i}} x_{n+1}-x_{n+1}\right\|\right) \\
& \quad=\left\|T_{r_{n+1}^{i} x_{n+1}}-T_{r_{n}^{i}} x_{n}\right\|\left(\left\|x_{n+1}-x_{n}\right\|+\frac{1}{r_{n+1}^{i}}\left|r_{n+1}^{i}-r_{n}^{i}\right|\left\|T_{r_{n+1}^{i}} x_{n+1}-x_{n+1}\right\|\right) \\
& \leq\left\|T_{r_{n+1}^{i}} x_{n+1}-T_{r_{n}^{i}} x_{n}\right\|\left(\left\|x_{n+1}-x_{n}\right\|+\frac{1}{a}\left|r_{n+1}^{i}-r_{n}^{i}\right|\left\|T_{r_{n+1}^{i}} x_{n+1}-x_{n+1}\right\|\right) .
\end{aligned}
$$

It follows that

$$
\left\|u_{n+1}^{i}-u_{n}^{i}\right\| \leq\left\|x_{n+1}-x_{n}\right\|+\frac{1}{a}\left|r_{n+1}^{i}-r_{n}^{i}\right|\left\|u_{n+1}^{i}-x_{n+1}\right\|
$$

for every $i=1,2, \ldots, N$. 
Substitute (3.11) into (3.5), we have

$$
\begin{aligned}
\left\|x_{n+1}-x_{n}\right\| \leq & \left(1-\alpha_{n}(1-\alpha)\right)\left(\sum_{i=1}^{N} \delta_{n}^{i}\left\|u_{n}^{i}-u_{n-1}^{i}\right\|+\sum_{i=1}^{N}\left|\delta_{n}^{i}-\delta_{n-1}^{i}\right|\left\|u_{n-1}^{i}\right\|\right) \\
& +\left|\alpha_{n}-\alpha_{n-1}\right|\left\|f\left(z_{n-1}\right)\right\|+\left(1-\alpha_{n}\right)\left\|S_{n}^{A} z_{n-1}-S_{n-1}^{A} z_{n-1}\right\| \\
& +\left|\alpha_{n-1}-\alpha_{n}\right|\left\|S_{n-1}^{A} z_{n-1}\right\| \\
\leq & \left(1-\alpha_{n}(1-\alpha)\right)\left(\sum_{i=1}^{N} \delta_{n}^{i}\left(\left\|x_{n+1}-x_{n}\right\|+\frac{1}{a}\left|r_{n+1}^{i}-r_{n}^{i}\right|\left\|u_{n+1}^{i}-x_{n+1}\right\|\right)\right. \\
& \left.+\sum_{i=1}^{N}\left|\delta_{n}^{i}-\delta_{n-1}^{i}\right|\left\|u_{n-1}^{i}\right\|\right) \\
& +\left|\alpha_{n}-\alpha_{n-1}\right| \mid f\left(z_{n-1}\right)\left\|+\left(1-\alpha_{n}\right)\right\| S_{n}^{A} z_{n-1}-S_{n-1}^{A} z_{n-1} \| \\
& +\left|\alpha_{n-1}-\alpha_{n}\right|\left\|S_{n-1}^{A} z_{n-1}\right\| \\
& +\left(1-\alpha_{n}(1-\alpha)\right)\left(\left\|x_{n+1}-x_{n}\right\|+\sum_{i=1}^{N} \delta_{n}^{i} \frac{1}{a}\left|r_{n+1}^{i}-r_{n}^{i}\right|\left\|u_{n+1}^{i}-x_{n+1}\right\|\right. \\
& +\sum_{i=1}^{N}\left|\delta_{n}^{i}-\delta_{n-1}^{i}\right|\left\|u_{n-1}^{i}\right\|+\left|\alpha_{n}-\alpha_{n-1}\right|\left\|f\left(z_{n-1}\right)\right\| \\
& +\left|\alpha_{n-1} z_{n-1}\left\|+\left|\alpha_{n-1}-\alpha_{n}\right|\right\| S_{n-1}^{A} z_{n-1} \| .\right. \\
& +\sum_{i=1}^{N}\left|\delta_{n}^{i}-\delta_{n-1}^{i}\right|\left\|S_{n-1}^{A} z_{n-1}\right\| \\
& +\left|\alpha_{n-1}-\alpha_{n-1}\right|\left\|f\left(z_{n-1}\right)\right\|+\left(1-\alpha_{n}\right)\left\|S_{n}^{A} z_{n-1}-S_{n-1}^{A} z_{n-1}\right\|
\end{aligned}
$$

By (3.12), (3.6), conditions (iii), (iv) and Lemma 2.3, we have

$$
\lim _{n \rightarrow \infty}\left\|x_{n+1}-x_{n}\right\|=0
$$

From (3.11), (3.13) and condition (iv), we have

$$
\lim _{n \rightarrow \infty}\left\|u_{n+1}^{i}-u_{n}^{i}\right\|=0, \quad \forall i=1,2, \ldots, N
$$

Let $p \in \mathbb{F}$. From $u_{n}^{i}=T_{r_{n}^{i}} x_{n}$ for every $i=1,2, \ldots, N$, we have

$$
\begin{aligned}
\left\|u_{n}^{i}-p\right\|^{2} & =\left\|T_{r_{n}^{i}} x_{n}-T_{r_{n}^{i}} p\right\|^{2} \\
& \leq\left\langle T_{r_{n}^{i}} x_{n}-T_{r_{n}^{i}} p, x_{n}-p\right\rangle \\
& =\frac{1}{2}\left(\left\|u_{n}^{i}-p\right\|^{2}+\left\|x_{n}-p\right\|^{2}-\left\|u_{n}^{i}-x_{n}\right\|^{2}\right) .
\end{aligned}
$$


It implies that

$$
\left\|u_{n}^{i}-p\right\|^{2} \leq\left\|x_{n}-p\right\|^{2}-\left\|u_{n}^{i}-x_{n}\right\|^{2} .
$$

By definition of $\left\{x_{n}\right\}$ and (3.15), we have

$$
\begin{aligned}
\left\|x_{n+1}-p\right\|^{2} & \leq \alpha_{n}\left\|f\left(z_{n}\right)-p\right\|^{2}+\left(1-\alpha_{n}\right)\left\|S_{n}^{A} z_{n}-p\right\|^{2} \\
& \leq \alpha_{n}\left\|f\left(z_{n}\right)-p\right\|^{2}+\left(1-\alpha_{n}\right)\left\|z_{n}-p\right\|^{2} \\
& =\alpha_{n}\left\|f\left(z_{n}\right)-p\right\|^{2}+\left(1-\alpha_{n}\right)\left\|\sum_{i=1}^{N} \delta_{n}^{i}\left(u_{n}^{i}-p\right)\right\|^{2} \\
& \leq \alpha_{n}\left\|f\left(z_{n}\right)-p\right\|^{2}+\left(1-\alpha_{n}\right) \sum_{i=1}^{N} \delta_{n}^{i}\left\|u_{n}^{i}-p\right\|^{2} \\
& \leq \alpha_{n}\left\|f\left(z_{n}\right)-p\right\|^{2}+\left(1-\alpha_{n}\right) \sum_{i=1}^{N} \delta_{n}^{i}\left(\left\|x_{n}-p\right\|^{2}-\left\|u_{n}^{i}-x_{n}\right\|^{2}\right) \\
& \leq \alpha_{n}\left\|f\left(z_{n}\right)-p\right\|^{2}+\left\|x_{n}-p\right\|^{2}-\left(1-\alpha_{n}\right) \sum_{i=1}^{N} \delta_{n}^{i}\left\|u_{n}^{i}-x_{n}\right\|^{2} .
\end{aligned}
$$

It implies that

$$
\begin{gathered}
\left(1-\alpha_{n}\right) \sum_{i=1}^{N} \delta_{n}^{i}\left\|u_{n}^{i}-x_{n}\right\|^{2} \leq \alpha_{n}\left\|f\left(z_{n}\right)-p\right\|^{2}+\left\|x_{n}-p\right\|^{2}-\left\|x_{n+1}-p\right\|^{2} \\
\leq \alpha_{n}\left\|f\left(z_{n}\right)-p\right\|^{2}+\left(\left\|x_{n}-p\right\|\right. \\
\left.+\left\|x_{n+1}-p\right\|\right)\left\|x_{n+1}-x_{n}\right\| .
\end{gathered}
$$

From conditions (i), (iii) and (3.13), we have

$$
\lim _{n \rightarrow \infty}\left\|u_{n}^{i}-x_{n}\right\|=0, \quad \forall i=1,2, \ldots, N
$$

Since

$$
x_{n+1}-S_{n}^{A} z_{n}=\alpha_{n}\left(f\left(z_{n}\right)-S_{n}^{A} z_{n}\right),
$$

from condition (i), we have

$$
\lim _{n \rightarrow \infty}\left\|x_{n+1}-S_{n}^{A} z_{n}\right\|=0
$$

From the definition of $z_{n}$, we have

$$
\begin{aligned}
\left\|z_{n}-x_{n}\right\| & =\left\|\sum_{i=1}^{N} \delta_{n}^{i}\left(u_{n}^{i}-x_{n}\right)\right\| \\
& \leq \sum_{i=1}^{N} \delta_{n}^{i}\left\|u_{n}^{i}-x_{n}\right\| .
\end{aligned}
$$


From condition (iii) and (3.17), we have

$$
\lim _{n \rightarrow \infty}\left\|z_{n}-x_{n}\right\|=0
$$

Since

$$
\left\|z_{n}-S_{n}^{A} z_{n}\right\| \leq\left\|z_{n}-x_{n}\right\|+\left\|x_{n}-x_{n+1}\right\|+\left\|x_{n+1}-S_{n}^{A} z_{n}\right\|,
$$

by (3.13), (3.18) and (3.19), we have

$$
\lim _{n \rightarrow \infty}\left\|z_{n}-S_{n}^{A} z_{n}\right\|=0 .
$$

Next, we show that

$$
\limsup _{n \rightarrow \infty}\left\langle f(z)-z, x_{n}-z\right\rangle \leq 0
$$

where $z=P_{\mathbb{F}} f(z)$. To show this inequality, take a subsequence $\left\{x_{n_{k}}\right\}$ of $\left\{x_{n}\right\}$ such that

$$
\limsup _{n \rightarrow \infty}\left\langle f(z)-z, x_{n}-z\right\rangle=\lim _{k \rightarrow \infty}\left\langle f(z)-z, x_{n_{k}}-z\right\rangle .
$$

Without loss of generality, we may assume that a subsequence $\left\{x_{n_{k}}\right\}$ of $\left\{x_{n}\right\}$ converges weakly to some $q \in H$. From (3.19), we have that $\left\{z_{n_{k}}\right\}$ converges weakly to $q$.

Since $\kappa<a \leq \alpha_{1}^{n, j}, \alpha_{2}^{n, j}, \alpha_{3}^{n, j} \leq b<1$ for all $j=1,2, \ldots, N$. Without loss of generality, we may assume that

$$
\begin{aligned}
\alpha_{1}^{n_{k}, j} & \rightarrow \alpha_{1}^{j} \in(\kappa, 1), \quad \alpha_{3}^{n_{k}, j} \rightarrow \alpha_{3}^{j} \in(\kappa, 1) \quad \text { and } \quad \alpha_{2}^{n_{k}, j} \rightarrow \alpha_{2}^{j} \in(\kappa, 1) \quad \text { as } k \rightarrow \infty, \\
\forall j & =1,2, \ldots, N .
\end{aligned}
$$

Let $S^{A}$ be the $S^{A}$-mapping generated by $S_{1}, S_{2}, \ldots, S_{N}, T_{1}, T_{2}, \ldots, T_{N}$ and $\beta_{1}, \beta_{2}, \ldots, \beta_{N}$, where $\beta_{j}=\left(\alpha_{1}^{j}, \alpha_{2}^{j}, \alpha_{3}^{j}\right), \forall j=1,2, \ldots, N$. By Lemma $2.8, S^{A}$ is a nonexpansive mapping, and $F\left(S^{A}\right)=\bigcap_{i=1}^{N} F\left(S_{i}\right) \cap \bigcap_{i=1}^{N} F\left(T_{i}\right)$.

By Lemma 2.9, we have

$$
\lim _{k \rightarrow \infty}\left\|S_{n_{k}}^{A} z_{n_{k}}-S^{A} z_{n_{k}}\right\|=0
$$

Since

$$
\left\|z_{n_{k}}-S^{A} z_{n_{k}}\right\| \leq\left\|z_{n_{k}}-S_{n_{k}}^{A} z_{n_{k}}\right\|+\left\|S_{n_{k}}^{A} z_{n_{k}}-S^{A} z_{n_{k}}\right\|
$$

by (3.20), (3.23), we have

$$
\lim _{k \rightarrow \infty}\left\|z_{n_{k}}-S^{A} z_{n_{k}}\right\|=0 .
$$

Since $\left\{z_{n_{k}}\right\}$ converges weakly to $q$ as $k \rightarrow \infty$ (3.24) and Lemma 2.7, we have

$$
q \in F\left(S^{A}\right)=\bigcap_{i=1}^{N} F\left(S_{i}\right) \cap \bigcap_{i=1}^{N} F\left(T_{i}\right) .
$$


Next, we show that $q \in \bigcap_{i=1}^{N} E P\left(F_{i}\right)$. To show this, we may assume that

$$
\lim _{k \rightarrow \infty} r_{n_{k}}^{i}=r^{i} \in[\theta, \eta], \quad \forall i=1,2, \ldots, N
$$

By Lemmas 2.5 and 2.6 , for every $i=1,2, \ldots, N$, we define $T_{r^{i}}: H \rightarrow C$ by

$$
T_{r^{i}}(x)=\left\{z \in C: F_{i}(z, y)+\frac{1}{r^{i}}\langle y-z, z-x\rangle \geq 0, \forall y \in C\right\}, \quad \forall x \in H \text { and } i=1,2, \ldots, N
$$

Then we have

$$
F_{i}\left(T_{r^{i}} x_{n}, y\right)+\frac{1}{r^{i}}\left\langle y-T_{r^{i}} x_{n}, T_{r^{i}} x_{n}-x_{n}\right\rangle \geq 0, \quad \forall y \in C \text { and } i=1,2, \ldots, N .
$$

From (3.1) and $u_{n}^{i}=T_{r_{n}^{i}} x_{n}$, we have

$$
F_{i}\left(T_{r_{n}^{i}} x_{n}, y\right)+\frac{1}{r_{n}^{i}}\left\langle y-T_{r_{n}^{i}} x_{n}, T_{r_{n}^{i}} x_{n}-x_{n}\right\rangle \geq 0, \quad \forall y \in C \text { and } i=1,2, \ldots, N
$$

It implies that

$$
F_{i}\left(T_{r^{i} x_{n_{k}}}, T_{r_{n_{k}}^{i}} x_{n_{k}}\right)+\frac{1}{r^{i}}\left\langle T_{r_{n_{k}}^{i}} x_{n_{k}}-T_{r^{i}} x_{n_{k}}, T_{r^{i} x_{n_{k}}}-x_{n_{k}}\right\rangle \geq 0, \quad \forall i=1,2, \ldots, N
$$

and

$$
F_{i}\left(T_{r_{n_{k}}^{i}} x_{n_{k}}, T_{r^{i}} x_{n_{k}}\right)+\frac{1}{r_{n_{k}}^{i}}\left\langle T_{r^{i}} x_{n_{k}}-T_{r_{n_{k}}^{i}} x_{n_{k}}, T_{r_{n_{k}}^{i}} x_{n_{k}}-x_{n_{k}}\right\rangle \geq 0, \quad \forall i=1,2, \ldots, N
$$

By (A2), we have

$$
\frac{1}{r^{i}}\left\langle T_{r_{n_{k}}^{i}} x_{n_{k}}-T_{r^{i} x_{n_{k}}}, T_{r^{i}} x_{n_{k}}-x_{n_{k}}\right\rangle+\frac{1}{r_{n_{k}}^{i}}\left\langle T_{r^{i}} x_{n_{k}}-T_{r_{n_{k}}^{i}} x_{n_{k}}, T_{r_{n_{k}}^{i}} x_{n_{k}}-x_{n_{k}}\right\rangle \geq 0 .
$$

It implies that

$$
\left\langle T_{r_{n_{k}}^{i}} x_{n_{k}}-T_{r^{i}} x_{n_{k}}, \frac{T_{r^{i}} x_{n_{k}}-x_{n_{k}}}{r^{i}}-\frac{T_{r_{n_{k}}^{i}} x_{n_{k}}-x_{n_{k}}}{r_{n_{k}}^{i}}\right\rangle \geq 0
$$

It follows that

$$
\left\langle T_{r_{n_{k}}^{i}} x_{n_{k}}-T_{r^{i} x_{n_{k}}}, T_{r^{i}} x_{n_{k}}-x_{n_{k}}-\frac{r^{i}}{r_{n_{k}}^{i}}\left(T_{r_{n_{k}}^{i}} x_{n_{k}}-x_{n_{k}}\right)\right\rangle \geq 0
$$

Then

$$
\begin{aligned}
0 & \leq\left\langle T_{r_{n_{k}}^{i}} x_{n_{k}}-T_{r^{i} x_{n_{k}}}, T_{r^{i}} x_{n_{k}}-T_{r_{n_{k}}^{i}} x_{n_{k}}+T_{r_{n_{k}}^{i}} x_{n_{k}}-x_{n_{k}}-\frac{r^{i}}{r_{n_{k}}^{i}}\left(T_{r_{n_{k}}^{i}} x_{n_{k}}-x_{n_{k}}\right)\right\rangle \\
& =\left\langle T_{r_{n_{k}}^{i}} x_{n_{k}}-T_{r^{i}} x_{n_{k}}, T_{r^{i}} x_{n_{k}}-T_{r_{n_{k}}^{i}} x_{n_{k}}+\left(1-\frac{r^{i}}{r_{n_{k}}^{i}}\right)\left(T_{r_{n_{k}}^{i}} x_{n_{k}}-x_{n_{k}}\right)\right\rangle .
\end{aligned}
$$


It follows that

$$
\begin{aligned}
\left\|T_{r_{n_{k}}^{i}} x_{n_{k}}-T_{r^{i}} x_{n_{k}}\right\|^{2} & \leq\left\langle T_{r_{n_{k}}^{i}} x_{n_{k}}-T_{r^{i}} x_{n_{k}},\left(1-\frac{r^{i}}{r_{n_{k}}^{i}}\right)\left(T_{r_{n_{k}}^{i}} x_{n_{k}}-x_{n_{k}}\right)\right\rangle \\
& \leq\left\|T_{r_{n_{k}}^{i}} x_{n_{k}}-T_{r^{i}} x_{n_{k}}\right\|\left|1-\frac{r^{i}}{r_{n_{k}}^{i}}\right|\left\|T_{r_{n_{k}}^{i}} x_{n_{k}}-x_{n_{k}}\right\| .
\end{aligned}
$$

It implies that

$$
\left\|T_{r_{n_{k}}^{i}} x_{n_{k}}-T_{r^{i}} x_{n_{k}}\right\| \leq \frac{1}{a}\left|r_{n_{k}}^{i}-r^{i}\right|\left\|T_{r_{n_{k}}^{i}} x_{n_{k}}-x_{n_{k}}\right\| .
$$

From $\lim _{k \rightarrow \infty} r_{n_{k}}^{i}=r^{i}$ and (3.17), we have

$$
\lim _{k \rightarrow \infty}\left\|T_{r_{n_{k}}^{i}} x_{n_{k}}-T_{r^{i}} x_{n_{k}}\right\|=0, \quad \forall i=1,2, \ldots, N .
$$

For every $i=1,2, \ldots, N$, we have

$$
\begin{aligned}
\left\|x_{n_{k}}-T_{r^{i}} x_{n_{k}}\right\| & \leq\left\|x_{n_{k}}-T_{r_{n_{k}}^{i}} x_{n_{k}}\right\|+\left\|T_{r_{n_{k}}^{i}} x_{n_{k}}-T_{r^{i}} x_{n_{k}}\right\| \\
& =\left\|x_{n_{k}}-u_{n_{k}}^{i}\right\|+\left\|T_{r_{n_{k}}^{i}} x_{n_{k}}-T_{r^{i}} x_{n_{k}}\right\|,
\end{aligned}
$$

by (3.17) and (3.26), we have

$$
\lim _{k \rightarrow \infty}\left\|x_{n_{k}}-T_{r^{i}} x_{n_{k}}\right\|=0, \quad \forall i=1,2, \ldots, N
$$

Since a subsequence $\left\{x_{n_{k}}\right\}$ of $\left\{x_{n}\right\}$ converges weakly to $q$ as $k \rightarrow \infty$, from (3.27) and Lemma 2.7, we have

$$
q \in F\left(T_{r^{i}}\right), \quad \forall i=1,2, \ldots, N .
$$

Then

$$
q \in \bigcap_{i=1}^{N} F\left(T_{r^{i}}\right)
$$

From Lemma 2.6, we have $E P\left(F_{i}\right)=F\left(T_{r^{i}}\right), \forall i=1,2, \ldots, N$. From (3.28), we have

$$
q \in \bigcap_{i=1}^{N} F\left(T_{r^{i}}\right)=\bigcap_{i=1}^{N} E P\left(F_{i}\right) .
$$

By (3.25) and (3.29), we have

$$
q \in \mathbb{F} .
$$

Since $x_{n_{k}} \rightarrow q$ as $k \rightarrow \infty$ and $q \in \mathbb{F}$ and (3.22), we have

$$
\limsup _{n \rightarrow \infty}\left\langle f(z)-z, x_{n}-z\right\rangle=\lim _{k \rightarrow \infty}\left\langle f(z)-z, x_{n_{k}}-z\right\rangle=\langle f(z)-z, q-z\rangle \leq 0 .
$$


Finally, we show that $\left\{x_{n}\right\}$ converges strongly to $z=P_{\mathbb{F}} f(z)$. Putting $z=P_{\mathbb{E}} f(z)$, by nonexpansiveness of $S^{A}$, we have

$$
\begin{aligned}
\left\|x_{n+1}-z\right\|^{2}= & \left\|\alpha_{n}\left(f\left(z_{n}\right)-z\right)+\left(1-\alpha_{n}\right)\left(S_{n}^{A} z_{n}-z\right)\right\|^{2} \\
\leq & \left(1-\alpha_{n}\right)^{2}\left\|S_{n}^{A} z_{n}-z\right\|^{2}+2 \alpha_{n}\left\langle f\left(z_{n}\right)-z, x_{n+1}-z\right\rangle \\
= & \left(1-\alpha_{n}\right)^{2}\left\|S_{n}^{A} z_{n}-z\right\|^{2}+2 \alpha_{n}\left\langle f\left(z_{n}\right)-f(z), x_{n+1}-z\right\rangle \\
& +2 \alpha_{n}\left\langle f(z)-z, x_{n+1}-z\right\rangle \\
\leq & \left(1-2 \alpha_{n}+\alpha_{n}^{2}\right)\left\|z_{n}-z\right\|^{2}+2 \alpha_{n} \alpha\left\|z_{n}-z\right\|\left\|x_{n+1}-z\right\| \\
& +2 \alpha_{n}\left\langle f(z)-z, x_{n+1}-z\right\rangle \\
\leq & \left(1-2 \alpha_{n}+\alpha_{n}^{2}\right)\left\|z_{n}-z\right\|^{2}+\alpha_{n} \alpha\left\|z_{n}-z\right\|^{2}+\alpha_{n} \alpha\left\|x_{n+1}-z\right\|^{2} \\
& +2 \alpha_{n}\left\langle f(z)-z, x_{n+1}-z\right\rangle \\
\leq & \left(1-2 \alpha_{n}+\alpha_{n} \alpha\right)\left\|x_{n}-z\right\|^{2}+\alpha_{n}^{2}\left\|x_{n}-z\right\|^{2}+\alpha_{n} \alpha\left\|x_{n+1}-z\right\|^{2} \\
& +2 \alpha_{n}\left\langle f(z)-z, x_{n+1}-z\right\rangle \\
= & \left(1-\alpha_{n} \alpha-2 \alpha_{n}(1-\alpha)\right)\left\|x_{n}-z\right\|^{2}+\alpha_{n}^{2}\left\|x_{n}-z\right\|^{2}+\alpha_{n} \alpha\left\|x_{n+1}-z\right\|^{2} \\
& +2 \alpha_{n}\left\langle f(z)-z, x_{n+1}-z\right\rangle .
\end{aligned}
$$

It implies that

$$
\begin{aligned}
\left\|x_{n+1}-z\right\|^{2} \leq & \left(1-\frac{2 \alpha_{n}(1-\alpha)}{1-\alpha_{n} \alpha}\right)\left\|x_{n}-z\right\|^{2}+\frac{\alpha_{n}^{2}}{1-\alpha_{n} \alpha}\left\|x_{n}-z\right\|^{2} \\
& +\frac{2 \alpha_{n}}{1-\alpha_{n} \alpha}\left\langle f(z)-z, x_{n+1}-z\right\rangle .
\end{aligned}
$$

This implies that by condition (i), (3.21) and Lemma 2.2, we have that the sequence $\left\{x_{n}\right\}$ converges strongly to $z=P_{\mathbb{F}} f(z)$. By (3.19), we have

$$
\left\|z_{n}-z\right\| \leq\left\|z_{n}-x_{n}\right\|+\left\|x_{n}-z\right\| \rightarrow 0 \quad \text { as } n \rightarrow \infty .
$$

This completes the proof.

\section{Applications}

In this section, we apply our main result to prove strong convergence theorems involving variational inclusion problems and variational inequality problems. To prove these results, we need definition and lemmas as follows.

A set-valued mapping $M: H \rightarrow 2^{H}$ is called monotone if for all $x, y \in H, f \in M x$ and $g \in M y$ imply that $\langle x-y, f-g\rangle \geq 0$. A monotone mapping $M: H \rightarrow 2^{H}$ is maximal if the graph $\operatorname{Graph}(M)$ of $M$ is not properly contained in the graph of any other monotone mapping. It is known that a monotone mapping $M$ is maximal if and only if for $(x, f) \in$ $H \times H,\langle x-y, f-g\rangle \geq 0$ for every $(y, g) \in \operatorname{Graph}(M)$ implies that $f \in M x$.

Next, we consider the following so-called variational inclusion problem: Find a $u \in H$ such that

$$
\theta \in B u+M u
$$


where $B: H \rightarrow H, M: H \rightarrow 2^{H}$ are two nonlinear mappings, and $\theta$ is zero vector in $H$ (see, for instance, [16-21]). The set of the solution of (4.1) is denoted by $V I(H, B, M)$.

Definition 4.1 (See [16]) Let $M: H \rightarrow 2^{H}$ be a multi-valued maximal monotone mapping, then the single-valued mapping $J_{M, \lambda}: H \rightarrow H$ defined by

$$
J_{M, \lambda}(u)=(I+\lambda M)^{-1}(u), \quad \forall u \in H,
$$

is called the resolvent operator associated with $M$, where $\lambda$ is any positive number, and $I$ is an identity mapping.

Lemma 4.1 (See [16]) $u \in H$ is a solution of variational inclusion (4.1) if and only if $u=$ $J_{M, \lambda}(u-\lambda B u), \forall \lambda>0$, i.e.,

$$
V I(H, B, M)=F\left(J_{M, \lambda}(I-\lambda B)\right), \quad \forall \lambda>0 .
$$

Further, if $\lambda \in(0,2 \alpha]$, then $V I(H, B, M)$ is a closed convex subset in $H$.

Lemma 4.2 (See [6]) The resolvent operator $J_{M, \lambda}$ associated with $M$ is single-valued, nonexpansive for all $\lambda>0$ and 1-inverse-strongly monotone.

A mapping $A$ of $C$ into $H$ is called $\alpha$-inverse strongly monotone, see [22], if there exists a positive real number $\alpha$ such that

$$
\langle x-y, A x-A y\rangle \geq \alpha\|A x-A y\|^{2}
$$

for all $x, y \in C$. The variational inequality problem is to find $u \in C$ such that

$$
\langle A u, v-u\rangle \geq 0
$$

for all $v \in C$. The set of solutions of the variational inequality is denoted by $\operatorname{VI}(C, A)$. We need the following lemma to prove a strong convergence theorem in this section.

Lemma 4.3 (See [23]) Let C be a closed convex subset of Hilbert space H. Let $A_{i}: C \rightarrow H$ be mappings, and let $G_{i}: C \rightarrow C$ be defined by $G_{i}(y)=P_{C}\left(I-\lambda_{i} A_{i}\right) y$ with $\lambda_{i}>0, \forall i=1,2, \ldots, N$. Then $x^{*} \in \bigcap_{i=1}^{N} V I\left(C, A_{i}\right)$ if and only if $x^{*} \in \bigcap_{i=1}^{N} F\left(G_{i}\right)$.

Theorem 4.4 Let $C$ be a nonempty closed convex subset of Hilbert spaces $H$, and let $f$ be an $\alpha$-contraction on $H$. For every $i=1,2, \ldots, N$, let $F_{i}$ be a bifunction from $C \times C$ into $\mathbb{R}$ satisfying (A1)-(A4), let $A_{i}: C \rightarrow H$ be an $\alpha_{i}$-inverse strongly monotone, and let $G_{i}: C \rightarrow C$ be a mapping defined by $G_{i}(y)=P_{C}\left(I-\lambda_{i} A_{i}\right) y, \forall y \in C$ with $\lambda_{i} \in(0,1] \subset\left(0,2 \alpha_{i}\right)$. Let $\left\{T_{i}\right\}_{i=1}^{N}$ be a finite family of $\kappa_{i}$-strict pseudo-contractions of $C$ into itself with $\mathbb{F} \equiv \bigcap_{i=1}^{N} V I\left(C, A_{i}\right) \cap$ $\bigcap_{i=1}^{N} F\left(T_{i}\right) \cap \bigcap_{i=1}^{N} E P\left(F_{i}\right) \neq \emptyset$ and $\kappa=\max \left\{\kappa_{i}: i=1,2, \ldots, N\right\}$, and let $\alpha_{j}^{(n)}=\left(\alpha_{1}^{n, j}, \alpha_{2}^{n, j}, \alpha_{3}^{n, j}\right) \in$ $I \times I \times I, j=1,2,3, \ldots, N$, where $I=[0,1], \alpha_{1}^{n, j}+\alpha_{2}^{n, j}+\alpha_{3}^{n, j}=1, \alpha_{1}^{n, j}, \alpha_{2}^{n, j}, \alpha_{3}^{n, j} \in[a, b] \subset(\kappa, 1)$ for all $j=1,2, \ldots, N$. Let $S_{n}^{A}$ be the $S^{A}$-mapping generated by $G_{1}, G_{2}, \ldots, G_{N}, T_{1}, T_{2}, \ldots, T_{N}$ 
and $\alpha_{1}^{(n)}, \alpha_{2}^{(n)}, \ldots, \alpha_{N}^{(n)}$. Let $\left\{x_{n}\right\}$ and $\left\{z_{n}\right\}$ be the sequences generated by $x_{1} \in C$ and

$$
\left\{\begin{array}{l}
F_{i}\left(u_{n}^{i}, y\right)+\frac{1}{r_{n}^{i}}\left\langle y-u_{n}^{i}, u_{n}^{i}-x_{n}\right\rangle \geq 0, \quad \forall y \in C \text { and } i=1,2, \ldots, N \\
z_{n}=\sum_{i=1}^{N} \delta_{n}^{i} u_{n}^{i}, \\
x_{n+1}=\alpha_{n} f\left(z_{n}\right)+\left(1-\alpha_{n}\right) S_{n}^{A} z_{n}, \quad \forall n \geq 1,
\end{array}\right.
$$

where $\left\{\alpha_{n}\right\}$ is a sequence in $[0,1]$. Assume that the following conditions hold:

(i) $\lim _{n \rightarrow \infty} \alpha_{n}=0, \sum_{n=1}^{\infty} \alpha_{n}=\infty$;

(ii) $\sum_{n=1}^{\infty}\left|\alpha_{1}^{n+1, j}-\alpha_{1}^{n, j}\right|<\infty, \sum_{n=1}^{\infty}\left|\alpha_{3}^{n+1, j}-\alpha_{3}^{n, j}\right|<\infty$ for all $j \in\{1,2,3, \ldots, N\}$ and $\sum_{n=1}^{\infty}\left|\alpha_{n+1}-\alpha_{n}\right|<\infty$

(iii) $\sum_{i=1}^{N} \delta_{n}^{i}=1, \sum_{n=1}^{\infty}\left|\delta_{n+1}^{i}-\delta_{n}^{i}\right|<\infty$ and $\lim _{n \rightarrow \infty} \delta_{n}^{i}=\delta_{i} \in(\kappa, 1)$ for every $i=1,2, \ldots, N$;

(iv) $\kappa<\theta \leq r_{n}^{i} \leq \eta$ for every $i=1,2, \ldots, N$ and $\sum_{n=1}^{\infty}\left|r_{n+1}^{i}-r_{n}^{i}\right|<\infty$.

Then the sequence $\left\{x_{n}\right\}$ converges strongly to $x^{*}=P_{\mathbb{F}} f\left(x^{*}\right)$.

Proof First, we show that $\left(I-\lambda_{i} A_{i}\right)$ is a nonexpansive mapping for every $i=1,2, \ldots, N$. For $x, y \in C$, we have

$$
\begin{aligned}
\left\|\left(I-\lambda_{i} A_{i}\right) x-\left(I-\lambda_{i} A_{i}\right) y\right\|^{2} & =\left\|x-y-\lambda_{i}\left(A_{i} x-A_{i} y\right)\right\|^{2} \\
& =\|x-y\|^{2}-2 \lambda_{i}\left\langle x-y, A_{i} x-A_{i} y\right\rangle+\lambda_{i}^{2}\left\|A_{i} x-A_{i} y\right\|^{2} \\
& \leq\|x-y\|^{2}-2 \alpha_{i} \lambda_{i}\left\|A_{i} x-A_{i} y\right\|^{2}+\lambda_{i}^{2}\left\|A_{i} x-A_{i} y\right\|^{2} \\
& =\|x-y\|^{2}+\lambda_{i}\left(\lambda_{i}-2 \alpha_{i}\right)\left\|A_{i} x-A_{i} y\right\|^{2} \\
& \leq\|x-y\|^{2} .
\end{aligned}
$$

Thus, $\left(I-\lambda_{i} A_{i}\right)$ is a nonexpansive mapping, and so is $G_{i}$ for all $i=1,2, \ldots, N$. Then we obtain the desired result from Lemma 4.3 and Theorem 3.1.

Corollary 4.5 Let $C$ be a nonempty closed convex subset of Hilbert spaces $H$, and let $f$ be an $\alpha$-contraction on $H$. For every $i=1,2, \ldots, N$, let $F_{i}$ be a bifunction from $C \times C$ into $\mathbb{R}$, satisfying (A1)-(A4), let $A_{i}: C \rightarrow H$ be an $\alpha_{i}$-inverse strongly monotone, and let $G_{i}$ : $C \rightarrow C$ be a mapping defined by $G_{i}(y)=P_{C}\left(I-\lambda_{i} A_{i}\right) y, \forall y \in C$ with $\lambda_{i} \in(0,1] \subset\left(0,2 \alpha_{i}\right)$. Let $\left\{T_{i}\right\}_{i=1}^{N}$ be a finite family of nonexpansive mappings of $C$ into itself with $\mathbb{F} \equiv \bigcap_{i=1}^{N} V I\left(C, A_{i}\right) \cap$ $\bigcap_{i=1}^{N} F\left(T_{i}\right) \cap \bigcap_{i=1}^{N} E P\left(F_{i}\right) \neq \emptyset$, and let $\alpha_{j}^{(n)}=\left(\alpha_{1}^{n, j}, \alpha_{2}^{n, j}, \alpha_{3}^{n, j}\right) \in I \times I \times I, j=1,2,3, \ldots, N$, where $I=[0,1], \alpha_{1}^{n, j}+\alpha_{2}^{n, j}+\alpha_{3}^{n, j}=1, \alpha_{1}^{n, j}, \alpha_{2}^{n, j}, \alpha_{3}^{n, j} \in[a, b] \subset(0,1)$ for all $j=1,2, \ldots, N$. Let $S_{n}^{A}$ be the $S^{A}$-mapping generated by $G_{1}, G_{2}, \ldots, G_{N}, T_{1}, T_{2}, \ldots, T_{N}$ and $\alpha_{1}^{(n)}, \alpha_{2}^{(n)}, \ldots, \alpha_{N}^{(n)}$. Let $\left\{x_{n}\right\}$ and $\left\{z_{n}\right\}$ be the sequences generated by $x_{1} \in C$ and

$$
\left\{\begin{array}{l}
F_{i}\left(u_{n}^{i}, y\right)+\frac{1}{r_{n}^{i}}\left\langle y-u_{n}^{i}, u_{n}^{i}-x_{n}\right\rangle \geq 0, \quad \forall y \in C \text { and } i=1,2, \ldots, N \\
z_{n}=\sum_{i=1}^{N} \delta_{n}^{i} u_{n}^{i}, \\
x_{n+1}=\alpha_{n} f\left(z_{n}\right)+\left(1-\alpha_{n}\right) S_{n}^{A} z_{n}, \quad \forall n \geq 1,
\end{array}\right.
$$

where $\left\{\alpha_{n}\right\}$ is a sequence in $[0,1]$. Assume that the following conditions hold:

(i) $\lim _{n \rightarrow \infty} \alpha_{n}=0, \sum_{n=1}^{\infty} \alpha_{n}=\infty$;

(ii) $\sum_{n=1}^{\infty}\left|\alpha_{1}^{n+1, j}-\alpha_{1}^{n, j}\right|<\infty, \sum_{n=1}^{\infty}\left|\alpha_{3}^{n+1, j}-\alpha_{3}^{n, j}\right|<\infty$ for all $j \in\{1,2,3, \ldots, N\}$ and $\sum_{n=1}^{\infty}\left|\alpha_{n+1}-\alpha_{n}\right|<\infty$; 
(iii) $\sum_{i=1}^{N} \delta_{n}^{i}=1, \sum_{n=1}^{\infty}\left|\delta_{n+1}^{i}-\delta_{n}^{i}\right|<\infty$ and $\lim _{n \rightarrow \infty} \delta_{n}^{i}=\delta_{i} \in(0,1)$ for every $i=1,2, \ldots, N$;

(iv) $0<\theta \leq r_{n}^{i} \leq \eta$ for every $i=1,2, \ldots, N$ and $\sum_{n=1}^{\infty}\left|r_{n+1}^{i}-r_{n}^{i}\right|<\infty$.

Then the sequence $\left\{x_{n}\right\}$ converges strongly to $x^{*}=P_{\mathbb{F}} f\left(x^{*}\right)$.

Proof Since $\left\{T_{i}\right\}_{i=1}^{N}$ is a finite family of nonexpansive mappings, we have that $\left\{T_{i}\right\}_{i=1}^{N}$ is a finite family of $\kappa_{i}$-strict pseudo-contractive mappings. From Theorem 4.4, we can draw the desired conclusion.

Theorem 4.6 Let $C$ be a nonempty closed convex subset of Hilbert spaces $H$, and let $f$ be an $\alpha$-contraction on $H$. For every $i=1,2, \ldots, N$, let $F_{i}$ be a bifunction from $C \times C$ into $\mathbb{R}$ satisfying (A1)-(A4). Let $M_{i}: H \rightarrow 2^{H}$ be maximal monotone mappings for every $i=$ $1,2, \ldots, N$, and let $B_{i}: H \rightarrow H$ be a $\delta_{i}$-inverse strongly monotone mapping for every $i=$ $1,2, \ldots, N$. Let $G_{i}: H \rightarrow H$ be a mapping defined by $J_{M_{i}, \eta}\left(I-\eta B_{i}\right) x=G_{i} x$ for every $x \in H$ with $\eta \in\left(0,2 \delta_{i}\right) i=1,2, \ldots, N$. Let $\left\{T_{i}\right\}_{i=1}^{N}$ be a finite family of $\kappa_{i}$-strict pseudo-contractions of $H$ into itself with $\mathbb{F} \equiv \bigcap_{i=1}^{N} V\left(H, B_{i}, M_{i}\right) \cap \bigcap_{i=1}^{N} F\left(T_{i}\right) \cap \bigcap_{i=1}^{N} E P\left(F_{i}\right) \neq \emptyset$ and $\kappa=\max \left\{\kappa_{i}\right.$ : $i=1,2, \ldots, N\}$, and let $\alpha_{j}^{(n)}=\left(\alpha_{1}^{n, j}, \alpha_{2}^{n, j}, \alpha_{3}^{n, j}\right) \in I \times I \times I, j=1,2,3, \ldots, N$, where $I=[0,1]$, $\alpha_{1}^{n, j}+\alpha_{2}^{n, j}+\alpha_{3}^{n, j}=1, \alpha_{1}^{n, j}, \alpha_{2}^{n, j}, \alpha_{3}^{n, j} \in[a, b] \subset(\kappa, 1)$ for all $j=1,2, \ldots, N$. Let $S_{n}^{A}$ be the $S^{A}-$ mapping generated by $G_{1}, G_{2}, \ldots, G_{N}, T_{1}, T_{2}, \ldots, T_{N}$ and $\alpha_{1}^{(n)}, \alpha_{2}^{(n)}, \ldots, \alpha_{N}^{(n)}$. Let $\left\{x_{n}\right\}$ and $\left\{z_{n}\right\}$ be the sequences generated by $x_{1} \in H$ and

$$
\left\{\begin{array}{l}
F_{i}\left(u_{n}^{i}, y\right)+\frac{1}{r_{n}^{i}}\left\langle y-u_{n}^{i}, u_{n}^{i}-x_{n}\right\rangle \geq 0, \quad \forall y \in C \text { and } i=1,2, \ldots, N, \\
z_{n}=\sum_{i=1}^{N} \delta_{n}^{i} u_{n}^{i}, \\
x_{n+1}=\alpha_{n} f\left(z_{n}\right)+\left(1-\alpha_{n}\right) S_{n}^{A} z_{n}, \quad \forall n \geq 1,
\end{array}\right.
$$

where $\left\{\alpha_{n}\right\}$ is a sequence in $[0,1]$. Assume that the following conditions hold:

(i) $\lim _{n \rightarrow \infty} \alpha_{n}=0, \sum_{n=1}^{\infty} \alpha_{n}=\infty$;

(ii) $\sum_{n=1}^{\infty}\left|\alpha_{1}^{n+1, j}-\alpha_{1}^{n, j}\right|<\infty, \sum_{n=1}^{\infty}\left|\alpha_{3}^{n+1, j}-\alpha_{3}^{n, j}\right|<\infty$ for all $j \in\{1,2,3, \ldots, N\}$ and $\sum_{n=1}^{\infty}\left|\alpha_{n+1}-\alpha_{n}\right|<\infty$

(iii) $\sum_{i=1}^{N} \delta_{n}^{i}=1, \sum_{n=1}^{\infty}\left|\delta_{n+1}^{i}-\delta_{n}^{i}\right|<\infty$ and $\lim _{n \rightarrow \infty} \delta_{n}^{i}=\delta_{i} \in(\kappa, 1)$, for every $i=1,2, \ldots, N$;

(iv) $\kappa<\theta \leq r_{n}^{i} \leq \eta$, for every $i=1,2, \ldots, N$ and $\sum_{n=1}^{\infty}\left|r_{n+1}^{i}-r_{n}^{i}\right|<\infty$.

Then the sequence $\left\{x_{n}\right\}$ converges strongly to $x^{*}=P_{\mathbb{F}} f\left(x^{*}\right)$.

Proof By using the same method as (4.4), we have that $I-\eta B_{i}$ is a nonexpansive mapping for every $i=1,2, \ldots, N$. By Lemma 4.2 , we have $J_{M_{i}, \eta}\left(I-\eta B_{i}\right)=G_{i}$ is a nonexpansive mapping for every $i=1,2, \ldots, N$. Then we obtain the desired result from Theorem 3.1.

\section{Example and numerical results}

In the last section, we give numerical examples to support our main results.

Example 5.1 Let $\mathbb{R}$ be the set of real numbers. For every $i=1,2, \ldots, N$, let the mappings $F_{i}: \mathbb{R} \times \mathbb{R} \rightarrow \mathbb{R}, T_{i}: \mathbb{R} \rightarrow \mathbb{R}, S_{i}: \mathbb{R} \rightarrow \mathbb{R}, f: \mathbb{R} \rightarrow \mathbb{R}$ defined by

$$
\begin{aligned}
& F_{i}(x, y)=i\left(4 y^{2}+x y-5 x^{2}\right), \\
& T_{i} x=(-1)^{2 i+1} \frac{3}{2} x,
\end{aligned}
$$




$$
\begin{aligned}
& S_{i} x=\frac{2 i}{2 i+1} x, \\
& f x=\frac{1}{3} x
\end{aligned}
$$

for every $x, y \in \mathbb{R}$.

Suppose that $S_{n}^{A}$ is the $S^{A}$-mapping generated by $S_{1}, S_{2}, \ldots, S_{N}, T_{1}, T_{2}, \ldots, T_{N}$ and $\alpha_{1}^{(n)}, \alpha_{2}^{(n)}, \ldots, \alpha_{N}^{(n)}$, where $\alpha_{j}^{(n)}=\left(\alpha_{1}^{(n, j)}, \alpha_{2}^{(n, j)}, \alpha_{3}^{(n, j)}\right)$ and $\alpha_{1}^{(n, j)}=\alpha_{2}^{(n, j)}=\alpha_{3}^{(n, j)}=\frac{1}{3}$ for every $n \geq 1$ and $j=1,2, \ldots, N$. Let the sequences $\left\{x_{n}\right\}$ and $\left\{z_{n}\right\}$ be generated by (3.1), where $\alpha_{n}=\frac{1}{5 n}$, $\delta_{n}^{i}=\left(\frac{1^{n}}{2^{i}}+\frac{1^{n}}{N \times 2^{N}}\right)$ and $r_{n}^{i}=\frac{i n}{n+1}$ for every $n \geq 1$ and $i=1,2, \ldots, N$. Then the sequences $\left\{x_{n}\right\}$ and $\left\{z_{n}\right\}$ converge strongly to 0 .

Solution. For every $i=1,2, \ldots, N$. It is easy to see that $S_{i}$ is nonexpansive and $T_{i}$ is $\frac{1}{5}$-strictly pseudo contractive mappings with $\{0\}=\bigcap_{i=1}^{N} F\left(S_{i}\right) \cap \bigcap_{i=1}^{N} F\left(T_{i}\right)$.

Since $S_{n}^{A}$ is the $S^{A}$-mapping generated by $S_{1}, S_{2}, \ldots, S_{N}, T_{1}, T_{2}, \ldots, T_{N}$ and $\alpha_{1}^{(n)}, \alpha_{2}^{(n)}$, $\ldots, \alpha_{N}^{(n)}$, where $\alpha_{j}^{(n)}=\left(\alpha_{1}^{(n, j)} \alpha_{2}^{(n, j)}, \alpha_{3}^{(n, j)}\right)$ and $\alpha_{1}^{(n, j)}=\alpha_{2}^{(n, j)}=\alpha_{3}^{(n, j)}=\frac{1}{3}$ for every $n \geq 1$ and $j=1,2, \ldots, N$, then we have

$$
\begin{aligned}
& U_{n, 0} x=x, \\
& U_{n, 1} x=\frac{2}{3}\left(\frac{1}{3} \times \frac{-3}{2} U_{n, 0}+\frac{1}{3} \times U_{n, 0}+\frac{1}{3}\right) x, \\
& U_{n, 2} x=\frac{4}{5}\left(\frac{1}{3} \times \frac{-3}{2} U_{n, 1}+\frac{1}{3} \times U_{n, 1}+\frac{1}{3}\right) x, \\
& U_{n, 3} x=\frac{6}{7}\left(\frac{1}{3} \times \frac{-3}{2} U_{n, 2}+\frac{1}{3} \times U_{n, 2}+\frac{1}{3}\right) x, \\
& \vdots \\
& U_{n, N-1} x=\frac{2(N-1)}{2(N-1)+1}\left(\frac{1}{3} \times \frac{-3}{2} U_{n, N-2}+\frac{1}{3} \times U_{n, N-2}+\frac{1}{3}\right) x, \\
& S_{n}^{A} x=U_{n, N} x=\frac{2 N}{2 N+1}\left(\frac{1}{3} \times \frac{-3}{2} U_{n, N-1}+\frac{1}{3} \times U_{n, N-1}+\frac{1}{3}\right) x
\end{aligned}
$$

for every $x \in \mathbb{R}$. From Lemma 2.8, we have $\{0\}=\bigcap_{i=1}^{N} F\left(S_{i}\right) \cap \bigcap_{i=1}^{N} F\left(T_{i}\right)=F\left(S_{n}^{A}\right)$. For every $n \geq 1$ and $i=1,2, \ldots, N$, we can see that $\sum_{i=1}^{N} \delta_{n}^{i}=\sum_{i=1}^{N}\left(\frac{1^{n}}{2^{i}}+\frac{1^{n}}{N \times 2^{N}}\right)=1$. From definition of $F_{i}$, we have $\bigcap_{i=1}^{N} E P\left(F_{i}\right)=\{0\}$. Then $\{0\}=\bigcap_{i=1}^{N} F\left(S_{i}\right) \cap \bigcap_{i=1}^{N} F\left(T_{i}\right) \cap \bigcap_{i=1}^{N} E P\left(F_{i}\right)=\mathbb{F}$.

For every $n \geq 1$ and $i=1,2, \ldots, N$, the mappings $F_{i}, T_{i}, S_{i}$ and $\alpha_{n}, r_{n}^{i}, \delta_{n}^{i}$ satisfy conditions in Theorem 3.1. Then from Theorem 3.1, we have the sequences $\left\{x_{n}\right\}$ and $\left\{z_{n}\right\}$ converge to 0 .

Next, we give numerical results to support this example. Let $r>0$ and $z \in \mathbb{R}$. For every $y \in \mathbb{R}$ and $i=1,2, \ldots, N$, and from Lemma 2.5 , there exist $x \in \mathbb{R}$ such that

$$
\begin{aligned}
& F_{i}(x, y)+\frac{1}{r}\langle y-x, x-z\rangle \geq 0 \\
& \quad \Leftrightarrow \quad i\left(4 y^{2}+x y-5 x^{2}\right)+\frac{1}{r}\langle y-x, x-z\rangle \geq 0 \\
& \quad \Leftrightarrow \quad 4 i r y^{2}+i r x y-5 i r x^{2}+(y-x)(x-z) \geq 0 \\
& \quad \Leftrightarrow \quad 4 i r y^{2}+i r x y-5 i r x^{2}+x y-x^{2}-z y+z x \geq 0 \\
& \quad \Leftrightarrow \quad 4 i r y^{2}+(\operatorname{rix}+x-z) y-\left(5 i r x^{2}+x^{2}-z x\right) \geq 0 .
\end{aligned}
$$


Put $G(y)=4 i r y^{2}+(r i x+x-z) y-\left(5 i r x^{2}+x^{2}-z x\right)$. Then $G$ is a quadratic function of $y$ with coefficient $a=4 i r, b=r i x+x-z, c=-\left(5 i r x^{2}+x^{2}-z x\right)$. Next, we compute the discriminant $\Delta$ of $G$ as follows:

$$
\begin{aligned}
\Delta & =b^{2}-4 a c \\
& =(r i x+x-z)^{2}+4(4 i r)\left(5 i r x^{2}+x^{2}-z x\right) \\
& =(r i x+x)^{2}-2 z(r i x+x)+z^{2}+80 i^{2} r^{2} x^{2}+16 i r x^{2}-16 i r z x \\
& =81 i^{2} r^{2} x^{2}+18 i r x^{2}+x^{2}-18 i r z x-2 z x+z^{2} \\
& =z^{2}+\left(81 i^{2} r^{2}+18 i r+1\right) x^{2}-2 z x(9 i r+1) \\
& =(z-x(9 i r+1))^{2} .
\end{aligned}
$$

Since $G(y) \geq 0$ for all $y \in \mathbb{R}$. If it has most one solution in $\mathbb{R}$, so $\Delta \leq 0$. It implies that $z=x(9$ ir +1$)$. Then we have

$$
x=T_{r} z=\frac{z}{i(9 r)+1}
$$

for all $r>0$ and $i=1,2, \ldots, N$. From (3.1) and (5.1), we have

$$
u_{n}^{i}=T_{r_{n}^{i}} x_{n}=\frac{x_{n}}{i\left(9 r_{n}^{i}\right)+1}
$$

for every $n \geq 1$ and $i=1,2, \ldots, N$. Since $\alpha_{n}=\frac{1}{5 n}, \delta_{n}^{i}=\left(\frac{1^{n}}{2^{i}}+\frac{1^{n}}{N \times 2^{N}}\right), r_{n}^{i}=\frac{i n}{n+1}$ and (5.2), we can rewrite (3.1) as follows:

$$
\left\{\begin{array}{l}
z_{n}=\sum_{i=1}^{N}\left(\frac{1^{n}}{2^{i}}+\frac{1^{n}}{N \times 2^{N}}\right) \frac{x_{n}}{i\left(9 \frac{i n}{n+1}\right)+1}, \\
x_{n+1}=\frac{1}{5 n} f\left(z_{n}\right)+\left(1-\frac{1}{5 n}\right) S_{n}^{A} z_{n}, \quad \forall n \geq 1
\end{array}\right.
$$

for every $n \geq 1$ and $i=1,2, \ldots, N$.

Put $N=8$ and initial points $x_{1}=700, x_{1}=-500$ in (5.3) we have the following results respectively.

The numerical results for initial points $x_{1}=700$ and $x_{1}=-500$ were shown in Tables 1 (Figure 1(b)) and 2 (Figure 1(a)), respectively. We observe that the sequences $\left\{x_{n}\right\}$ and $\left\{z_{n}\right\}$ converge to $0 \in \bigcap_{i=1}^{N} F\left(S_{i}\right) \cap \bigcap_{i=1}^{N} F\left(T_{i}\right) \cap \bigcap_{i=1}^{N} E P\left(F_{i}\right)$.

Table 1 The values of $\left\{z_{n}\right\}$ and $\left\{x_{n}\right\}$ with initial points $x_{1}=700, n=8$ and $N=8$

\begin{tabular}{lrr}
\hline $\boldsymbol{n}$ & \multicolumn{1}{l}{$\boldsymbol{z}_{\boldsymbol{n}}$} & \multicolumn{1}{c}{$\boldsymbol{x}_{\boldsymbol{n}}$} \\
\hline 1 & 75.9495089241 & 700.0000000000 \\
2 & 1.8273618170 & 21.5559869697 \\
3 & 0.0387720272 & 0.5073319122 \\
4 & 0.0007709831 & 0.0106843198 \\
5 & 0.0000147261 & 0.0002116628 \\
6 & 0.0000002736 & 0.0000040337 \\
7 & 0.0000000050 & 0.0000000748 \\
8 & 0.0000000001 & 0.0000000014 \\
\hline
\end{tabular}


Table 2 The values of $\left\{z_{n}\right\}$ and $\left\{x_{n}\right\}$ with initial points $x_{1}=-500, n=8$ and $N=8$

\begin{tabular}{llr}
\hline $\boldsymbol{n}$ & \multicolumn{1}{l}{$\boldsymbol{z}_{\boldsymbol{n}}$} & \multicolumn{1}{l}{$\boldsymbol{x}_{\boldsymbol{n}}$} \\
\hline 1 & -54.2496492315 & -500.0000000000 \\
2 & -1.3052584407 & -15.3971335498 \\
3 & -0.0276943051 & -0.3623799373 \\
4 & -0.0005507022 & -0.0076316570 \\
5 & -0.0000105186 & -0.0001511877 \\
6 & -0.0000001955 & -0.0000028812 \\
7 & -0.0000000036 & -0.0000000535 \\
8 & -0.0000000001 & -0.0000000010 \\
\hline
\end{tabular}

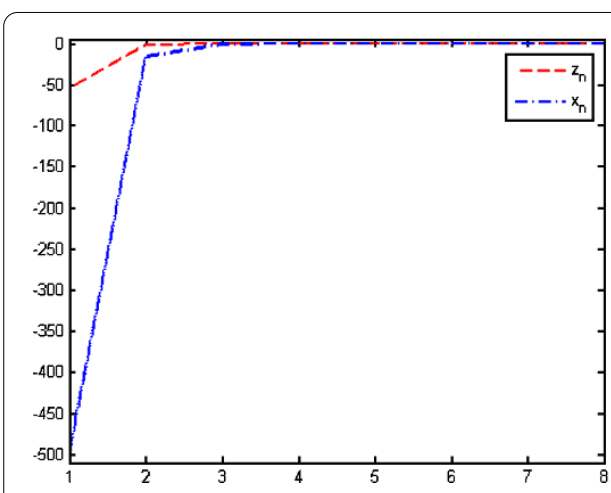

(a) $x_{1}=-500$

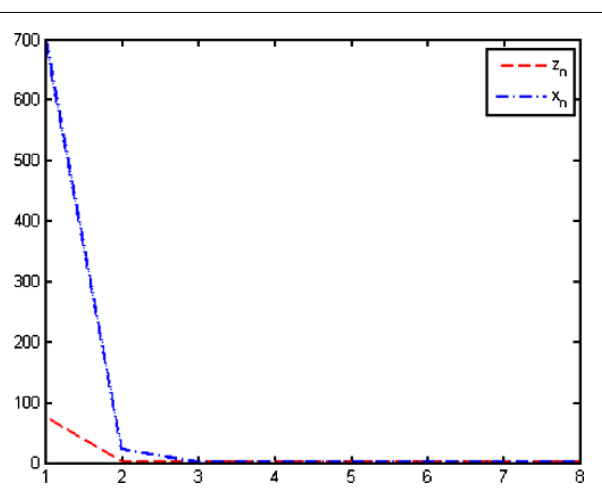

(b) $x_{1}=700$

Figure 1 The convergence comparison with different initial values (a) $x_{1}=-500$ and (b) $x_{1}=700$.

\section{Competing interests}

The author declares that they have no competing interests.

\section{Acknowledgements}

This research was supported by the Research Administration Division of King Mongkut's Institute of Technology Ladkrabang.

\section{Received: 28 March 2013 Accepted: 30 September 2013 Published: 09 Nov 2013}

\section{References}

1. Browder, FE, Petryshyn, WV: Construction of fixed points of nonlinear mappings in Hilbert space. J. Math. Anal. Appl. 20, 197-228 (1967)

2. Blum, E, Oettli, W: From optimization and variational inequalities to equilibrium problems. Math. Stud. 63, 123-145 (1994)

3. Combettes, PL, Hirstoaga, A: Equilibrium programming in Hilbert spaces. J. Nonlinear Convex Anal. 6, 117-136 (2005)

4. Moudafi, A, Théra, M: Proximal and dynamical approaches to equilibrium problems. In: II-Posed Variational Problems and Regularization Techniques. Lecture Notes in Economics and Mathematical Systems, vol. 477, pp. 187-201. Springer, Berlin (1999)

5. Kangtunyakarn, A, Suantai, S: Hybrid iterative scheme for generalized equilibrium problems and fixed point problems of finite family of nonexpansive mappings. Nonlinear Anal. Hybrid Syst. 3, 296-309 (2009)

6. Chang, SS, Lee, HWJ, Chan, CK: A new method for solving equilibrium problem fixed point problem and variational inequality problem with application to optimization. Nonlinear Anal. 70, 3307-3319 (2009)

7. Takahashi, S, Takahashi, W: Viscosity approximation methods for equilibrium problems and fixed point problems in Hilbert spaces. J. Math. Anal. Appl. 331, 506-515 (2007)

8. Takahashi, S, Takahashi, W: Viscosity approximation methods for equilibrium problems and fixed point problems in Hilbert spaces. J. Math. Anal. Appl. 331(1), 506-515 (2007)

9. Kangtunyakarn, A, Suantai, S: Strong convergence of a new iterative scheme for a finite family of strict pseudo-contractions. Comput. Math. Appl. 60, 680-694 (2010)

10. Takahashi, W: Nonlinear Functional Analysis. Yokohama Publishers, Yokohama (2000)

11. Xu, HK: Iterative algorithms for nonlinear operators. J. Lond. Math. Soc. 66, 240-256 (2002)

12. Xu, HK: An iterative approach to quadratic optimization. J. Optim. Theory Appl. 116, 659-678 (2003)

13. Marino, G, Xu, HK: Weak and strong convergence theorems for strict pseudo-contractions in Hilbert spaces. J. Math. Anal. Appl. 329, 336-346 (2007)

14. Combettes, PL, Hirstoaga, SA: Equilibrium programming in Hilbert spaces. J. Nonlinear Convex Anal. 6(1), 117-136 (2005) 
15. Browder, FE: Nonlinear operators and nonlinear equations of evolution in Banach spaces. Proc. Symp. Pure Math. 18, 78-81 (1976)

16. Zhang, SS, Lee, JHW, Chan, CK: Algorithms of common solutions for quasi variational inclusion and fixed point problems. Appl. Math. Mech. 29, 571-581 (2008)

17. Chang, SS: Set-valued variational inclusions in Banach spaces. J. Math. Anal. Appl. 248, 438-454 (2000)

18. Noor, MA, Noor, Kl: Sensitivity analysis for quasi-variational inclusions. J. Math. Anal. Appl. 236, $290-299$ (1999)

19. Takahashi, W, Toyoda, M: Weak convergence theorems for nonexpansive mappings and monotone mappings. J. Optim. Theory Appl. 118, 417-428 (2003)

20. Li, Y, Wu, C: On the convergence for an iterative method for quasivariational inclusions. Fixed Point Theory Appl. 2010, Article ID 278973 (2010). doi:10.1155/2010/278973

21. Hao, Y: On variational inclusion and common fixed point problems in Hilbert spaces with applications. Appl. Math. Comput. 217(7), 3000-3010 (2010)

22. liduka, $\mathrm{H}$, Takahashi, W: Weak convergence theorem by Cesáro means for nonexpansive mappings and inverse-strongly monotone mappings. J. Nonlinear Convex Anal. 7, 7105-7113 (2006)

23. Kangtunyakarn, A: Strong convergence theorem for a generalized equilibrium problem and system of variational inequalities problem and infinite family of strict pseudo-contractions. Fixed Point Theory Appl. 2011, 23 (2011)

10.1186/1687-1812-2013-295

Cite this article as: Kangtunyakarn: Iterative approximation of common element of solution sets of various nonlinear operator problems. Fixed Point Theory and Applications 2013, 2013:295

\section{Submit your manuscript to a SpringerOpen ${ }^{\circ}$ journal and benefit from:}

- Convenient online submission

- Rigorous peer review

- Immediate publication on acceptance

- Open access: articles freely available online

- High visibility within the field

- Retaining the copyright to your article 